\title{
Propagation of a curved weak shock
}

\author{
By A. MONICA AND PHOOLAN PRASAD \\ Department of Mathematics, Indian Institute of Science, Bangalore-560012 \\ e-mail: prasad@math.iisc.ernet.in
}

(Received 7 September 1999 and in revised form 3 August 2000)

Propagation of a curved shock is governed by a system of shock ray equations which is coupled to an infinite system of transport equations along these rays. For a twodimensional weak shock, it has been suggested that this system can be approximated by a hyperbolic system of four partial differential equations in a ray coordinate system, which consists of two independent variables $(\xi, t)$ where the curves $t=$ constant give successive positions of the shock and $\xi=$ constant give rays. The equations show that shock rays not only stretch longitudinally due to finite amplitude on a shock front but also turn due to a non-uniform distribution of the shock strength on it. These changes finally lead to a modification of the amplitude of the shock strength. Since discontinuities in the form of kinks appear on the shock, it is necessary to study the problem by using the correct conservation form of these equations. We use such a system of equations in conservation form to construct a total-variation-bounded finite difference scheme. The numerical solution captures converging shock fronts with a pair of kinks on them - the shock front emerges without the usual folds in the caustic region. The shock strength, even when the shock passes through the caustic region, remains so small that the small-amplitude theory remains valid. The shock strength ultimately decays with a well-defined geometrical shape of the shock front-a pair of kinks which separate a central disc from a pair of wings on the two sides. We also study the ultimate shape and decay of shocks of initially periodic shapes and plane shocks with a dent and a bulge.

\section{Introduction}

The propagation of a curved shock governed by a system of conservation laws is a complex phenomenon due to three important physical processes which take place simultaneously. These are (i) different points of the front travel with different speeds along rays, depending on the amplitude at these points, leading to a longitudinal stretching of the rays; (ii) a lateral deviation in the direction of a normal to the shock front, i.e. that of a ray, takes place due to non-uniform distribution of the shock strength on the shock; and (iii) the waves which catch up with the shock front from behind not only modify the shock motion but are themselves modified after getting reflected from it. Due to the first two processes, a smooth concave shock front with continuous amplitude distribution on it develops a pair of kinks after some time. Across the kink, the shock strength and the direction of the normal to the shock change discontinuously. A simple heuristic (approximate) theory for the propagation of curved shocks (Whitham 1974) shows that there are nonlinear waves propagating on a shock front itself, as a result of which kinks (called shock-shocks by Whitham) are formed. Formation of a kink and its propagation on a shock front was experimentally captured by Sturtevant \& Kulkarni (1976). For a weak shock (but not 
so weak that it follows the linear theory of acoustics), rays of the converging shock front curve away from the focal region and the shock front emerges flattened, with no loop. A kink on a shock front has been interpreted by Whitham as a trace of triple shock interaction, some understanding of which has emerged from experiments and computation of full gas dynamic equations, as well as a model equation called the two-dimensional Burgers equation (Collela \& Henderson 1990; Brio \& Hunter 1992, 1994; Tabak \& Rosales 1994). We discuss the relation between the results obtained from the two-dimensional Burgers equation with our results in this paper in $\S 6$.

Kinks appear not only on a shock front but also on each one of a one-parameter family of weakly nonlinear wavefronts in the high-frequency approximation (Prasad 1993, 1994; Prasad \& Sangeeta 1999). Unlike a shock front, the solution is continuous across a nonlinear wavefront and the governing equations are much simpler. Because of the formulation of the weakly nonlinear ray theory (WNLRT) by Morton, Prasad \& Ravindran (1992) and due to the availability of modern and highly sophisticated numerical methods for solutions of conservation laws, the work of Prasad \& Sangeeta shows that the computation of successive positions of a nonlinear wavefront with a kink is now a routine task. These conservation forms play an important role in this paper also.

Kevlahan (1996) studied weak shock propagation in non-uniform flows by using the shock ray equations together with an approximate form of the compatibility conditions for shock strength and the normal derivative of a physical variable behind the shock (following Ravindran \& Prasad 1993, also see Prasad 1993). But the solution could be easily obtained only till the critical time when the kinks form on the shock front. For a short time after the formation of kinks, he was able to extend the solution by propagating the shock disc and wing as separate fronts. The position of the kink at a later time was given by the intersection of the two parts. But this method gives only a rough estimate of the geometry and strength of the shock, which may change considerably over time. Therefore, to study the propagation of kinks over a large interval of time, one needs a physically realistic conservation form of these equations so that we can use available numerical schemes of conservation laws for capturing the kinks automatically and for obtaining the long-term behaviour of the solutions. It is simple to show that the intersection method used by Kevlahan gives a kink position which is the same as that obtained by solving the conservation laws used in this paper. A detailed proof of this is available in Prasad (2001). Therefore, the solutions obtained by Kevlahan are also the solutions of these conservation laws except for errors in numerical computation. Kevlahan has shown that the results of this theory agree very well with the experimental results of Sturtevant \& Kulkarni (1976) and with the numerical solution of the original gas dynamic equations. In fact, the comparison of the geometric shape of the shock front and kink position obtained by the NTSD (new theory of shock dynamics, that we have used in this paper) and those by the numerical solution of Euler's equations is presented in figure 4 of Kevlahan's paper. Since the two results overlap completely, we conclude that kinks actually propagate at the speed predicted by the conservation laws (3.12) and (3.13) in this paper. The results of the NTSD also agree completely with the experimental results (Kevlahan 1996, figure 7). This has prompted us not to question the correctness of the results of the NTSD and we concentrate in this paper on obtaining the long-term behaviour of a shock front with kinks and other minute details quantitatively. Apart from these numerical comparisons with the Euler solution and experimental results, following Giles, Prasad \& Ravindran (1996), we shall prove in $\S 3$ that the kinematic conservation laws (3.12) and (3.13), true for any propagating curve, represent conservation of the vector 
distance traversed by the kink (the meaning of which shall be explained along with the proof).

In this paper, we first present a derivation of the weak shock ray equations from WNLRT, a physically realistic conservation form of NTSD equations and finally, some results of numerical solutions of these equations for converging shock fronts starting from various kinds of initial geometry. Distributions of the shock strength and the normal derivative on these initial shock fronts have been varied in order to bring out some interesting results. A total-variation-bounded finite difference scheme based on the Lax-Friedrichs flux has been used to solve the system of conservation laws and Strang splitting is used to take into account the effects of the source term that appears in one of the equations. Effects of changing the initial strength of the shock or that of the normal derivative, and the effect of initial curvature on the formation, propagation and separation of kinks have been studied. Then we have studied the ultimate shape and decay of shocks with initially periodic shapes and plane shocks with a dent and bulge, and interpreted these results as corrugational stability of a shock front. Finally we have presented a comparison of our results with those obtained from other theories.

In order that we can appreciate the results on the resolution of a caustic in this paper, we present here the details of a representative case of the caustic formed by the linear wavefront of the two-dimensional wave equation with wave speed unity and with initial position of the front given in $\S 5.1$, case (i). We take here $b=4$ and $z=2$, i.e. the initial position of the wavefront is given by

$$
y^{2}=4 x \text { for } 0 \leqslant x \leqslant 1 \text { and } y= \pm(x+1) \text { for } x>1 \text {, }
$$

which consists of a central part which is a parabola and two wings on both sides of it. The wings are simply the two tangents to the parabola at the points $( \pm 2,1)$. It is interesting to note that the two branches of the caustic starting from the arete $(2,0)$ end at $(5, \pm 2)$ as shown in figure 1 . However, the linear wavefront has the usual fold not only in the region bounded by the two branches of the caustic, but also after it has crossed the end points of the two branches at $t=4 \sqrt{2}$ even though there is no caustic for $t>4 \sqrt{2}$. The persistence of the cusp type of singularity on the wavefront is due to the discontinuities in the curvature of the initial wavefront at $( \pm 2,1)$.

\section{Governing equations of the NTSD}

A system of shock ray equations consists of the ray equations derived from a shock manifold partial differential equation (or eikonal equation for a shock front, Prasad 1982) and an infinite system of compatibility conditions along a shock ray (Grinfel'd 1978; Maslov 1980; Anile \& Russo 1986; Srinivasan \& Prasad 1985). These compatibility conditions are derived from the equations governing the motion of the medium in which the shock propagates. It is interesting to note that unlike the wellknown geometrical optics theory for the propagation of a one-parameter family of wavefronts across which wave amplitude is continuous, the shock ray theory with an infinite system of compatibility conditions is exact. This is because geometrical optics requires a high-frequency approximation, which is satisfied exactly for a shock front. The explicit form of the first two compatibility conditions for a two-dimensional curved shock front in a polytropic gas has been derived by Ravindran \& Prasad (1993). These equations, even with just two compatibility conditions, are so complex that not much progress in actual computation of a shock propagation could be made (for some numerical results, see Singh \& Singh 1999). Under suitable assumptions, 


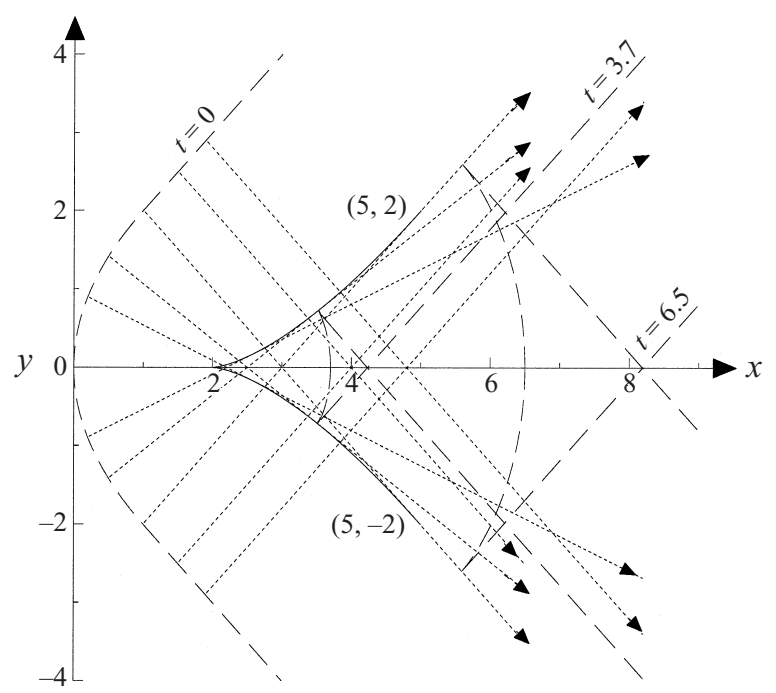

FIGURE 1. Linear wavefront propagation in an isotropic homogeneous medium with speed of propagation unity. Broken curves: wavefront; solid lines: caustic; dotted lines: rays.

these equations for a weak shock reduce to a rather simpler set (equations (5.73)-(5.77) of Prasad 1993), which we shall use in this paper.

The derivation of the equations of the NTSD for a weak shock (we simply call it NTSD omitting 'for a weak shock' henceforth in this paper) described in the previous paragraph, is quite clear but requires extremely complex algebraic calculations. Hence we first present one more derivation (Prasad 2000) from the equations of weakly nonlinear ray theory (Prasad 1993, 1994; Prasad \& Sangeeta 1999) simply denoted by WNLRT, derived under short-wave or high-frequency assumptions. Consider a weak shock front propagating into a polytropic gas at rest ahead of it. Then the shock will be followed by a one-parameter family of nonlinear waves belonging to the same characteristic field (or mode). Each one of these wavefronts will catch up with the shock, interact with it and then disappear. A nonlinear wave, while interacting with the shock, will be instantaneously coincident with it in the short-wave assumption. The ray equations of the WNLRT in three space dimensions for a particular nonlinear wavefront are

$$
\frac{\mathrm{d} \boldsymbol{x}}{\mathrm{d} t}=\boldsymbol{n} a_{0}\left(1+\epsilon \frac{\gamma+1}{2} w\right)
$$

and

$$
\frac{\mathrm{d} \boldsymbol{n}}{\mathrm{d} t}=-\epsilon \frac{\gamma+1}{2} a_{0} \mathbf{L} w,
$$

where $\boldsymbol{x}$ is the position of a nonlinear ray at time $t, \boldsymbol{n}$ is the unit normal of the wavefront (i.e. the direction of the ray), $\gamma$ is the ratio of the specific heats, $\mathbf{L}$ is a vector operator

$$
\mathbf{L}=\nabla-\boldsymbol{n}\langle\boldsymbol{n} \cdot \nabla\rangle
$$

each component of which represents a tangential derivative to the wavefront, $a_{0}$ is the constant sound velocity in the medium in which small-amplitude waves are propagating and $\epsilon w$ represents the amplitude of the wave in terms of which pertubations 
due to the waves in the density $\rho$, fluid velocity $\boldsymbol{q}$ and pressure $p$ are given by

$$
\rho-\rho_{0}=\epsilon \rho_{0} w, \quad \boldsymbol{q}=\epsilon \boldsymbol{n} a_{0} w, \quad p-p_{0}=\epsilon \rho_{0} a_{0}^{2} w .
$$

The transport equation for the amplitude $w$ on the nonlinear wavefront is

$$
\frac{\mathrm{d} w}{\mathrm{~d} t}=-\frac{1}{2} a_{0}\langle\nabla \cdot \boldsymbol{n}\rangle w
$$

where

$$
\frac{\mathrm{d}}{\mathrm{d} t}=\frac{\partial}{\partial t}+a_{0}\left(1+\epsilon \frac{\gamma+1}{2} w\right)\langle\boldsymbol{n} \cdot \nabla\rangle .
$$

Now we use the following theorem (Prasad 1993, p. 74):

THEOREM. For a weak shock, the shock ray velocity components are equal to the mean of the bicharacteristic velocity components just ahead of and just behind the shock, provided we take the wavefronts generating the characteristic surfaces ahead of and behind to be instantaneously coincident with the shock surface. Similarly, the rate of turning of the shock front is equal to the mean of the rates of turning of such wavefronts just ahead of and just behind the shock.

We denote the unit normal to the shock front by $\boldsymbol{N}$. For the linear wavefront just ahead of the shock and instantaneously coincident with it (this is actually a linear wavefront moving with the ray velocity $N$ multiplied by the local sound velocity $\left.a_{0}\right), w=0$ and the bicharacteristic velocity is $N a_{0}$. For the nonlinear wavefront just behind the shock and instantaneously coincident with it, we denote the amplitude $w$ by $\mu$. Then $\mu$ is the shock amplitude of the weak shock under consideration. Using the theorem and the results (2.1) and (2.2) with $\boldsymbol{n}=\boldsymbol{N}$, we get for a point $\boldsymbol{X}$ on the shock ray

$$
\begin{gathered}
\frac{\mathrm{d} \boldsymbol{X}}{\mathrm{d} T}=\frac{1}{2}\left\{a_{0} \boldsymbol{N}+\boldsymbol{N} a_{0}\left(1+\epsilon \frac{\gamma+1}{2} \mu\right)\right\}=N a_{0}\left(1+\epsilon \frac{\gamma+1}{4} \mu\right), \\
\frac{\mathrm{d} \boldsymbol{N}}{\mathrm{d} T}=-\frac{1}{2}\left\{0+\epsilon \frac{\gamma+1}{2} a_{0} \mathbf{L} \mu\right\}=-\epsilon \frac{\gamma+1}{4} a_{0} \mathbf{L} \mu,
\end{gathered}
$$

where $T$ is the time measured while moving along a shock ray. We take $w=\mu$ and $\boldsymbol{n}=\boldsymbol{N}$ in (2.5) and write it as

$$
\begin{aligned}
\frac{\mathrm{d} \mu}{\mathrm{d} T} & \equiv\left\{\frac{\partial}{\partial t}+a_{0}\left(1+\epsilon \frac{\gamma+1}{4} \mu\right)\langle\boldsymbol{N} \cdot \nabla\rangle\right\} \mu \\
& =-\frac{1}{2} a_{0}\langle\nabla \cdot \boldsymbol{N}\rangle \mu-\epsilon \frac{\gamma+1}{4} \mu\langle\boldsymbol{N} \cdot \nabla\rangle w,
\end{aligned}
$$

where we note that since $\mu$ is defined only on the shock front (and also on the instantaneously coincident nonlinear wavefront behind it but not on the other members of the one-parameter family of wavefronts following it), the normal derivative $\langle\boldsymbol{N} \cdot \nabla\rangle \mu$ does not make sense mathematically. We introduce a new variable, defined on the shock front

$$
\mu_{1}=\left.\epsilon\{\langle\boldsymbol{N} \cdot \nabla\rangle w\}\right|_{\text {shock front }},
$$

where $\epsilon$ appears to make $\mu_{1}=O(1)$ since we wish to consider variation of $w$ on a length scale over which the fast variable $\theta$ (introduced in the derivation of (2.5), see Prasad 2000) varies. This variable is $\theta=\phi / \epsilon$, where $\phi$ satisfies the characteristic partial differential equation $\phi_{t}+\langle\boldsymbol{q} \cdot \nabla \phi\rangle+a|\nabla \phi|=0$. 
The equation (2.9) leads to the first compatibility condition along a shock ray:

$$
\frac{\mathrm{d} \mu}{\mathrm{d} T}=\Omega_{s} \mu-\frac{\gamma+1}{4} \mu \mu_{1}
$$

where

$$
\bar{\Omega}_{s}=-\frac{1}{2} a_{0}\langle\nabla \cdot N\rangle
$$

is the value of the mean curvature of the nonlinear wavefront instantaneously coincident with the shock from behind.

To find the second compatibility condition along a shock, we differentiate $(2.5)$ in the direction of $\boldsymbol{n}$ but on the length scale over which $\theta$ varies. On this length scale, $\boldsymbol{n}$ is constant and we get after rearranging some terms

$$
\begin{gathered}
\left\{\frac{\partial}{\partial t}+a_{0}\left(1+\epsilon \frac{\gamma+1}{4} w\right)\langle\boldsymbol{n} \cdot \nabla\rangle\right\}\langle\boldsymbol{n} \cdot \nabla\rangle w=-\frac{1}{2}\langle\nabla \cdot \boldsymbol{n}\rangle\langle\boldsymbol{n} \cdot \nabla\rangle w-\epsilon \frac{\gamma+1}{4} w\langle\boldsymbol{n} \cdot \nabla\rangle^{2} w \\
-\epsilon \frac{\gamma+1}{4}\{\langle\boldsymbol{n} \cdot \nabla\rangle w\}^{2}
\end{gathered}
$$

Writing this equation for the wavefront instantaneously coincident with the shock, multiplying it by $\epsilon$ and introducing a variable $\mu_{2}$ by

$$
\mu_{2}=\left.\epsilon^{2}\left\{\langle\boldsymbol{n} \cdot \nabla\rangle^{2} w\right\}\right|_{\text {shock front }}
$$

we get

$$
\frac{\mathrm{d} \mu_{1}}{\mathrm{~d} T}=\bar{\Omega}_{s} \mu_{1}-\frac{\gamma+1}{4} \mu_{1}^{2}-\frac{\gamma+1}{4} \mu \mu_{2},
$$

which is the second compatibility condition along shock rays given by (2.7) and (2.8).

Similarly, higher-order compatibility conditions can be derived. Thus, for the Euler equations, we have derived the infinite system of compatibility conditions for a weak shock just from the dominant terms of WNLRT. Since the shock ray theory (SRT) can be derived from the WNLRT, the latter is more general than SRT. However, the results obtained by SRT are quantitatively different from those by WNLRT (see $\S 6.3)$.

As we have already mentioned, the shock ray theory is an exact theory (the weak shock assumption is another independent assumption) but since there is an infinite number of compatibility conditions on it, it is impossible to use it for computing shock propagation. Prasad \& Ravindran proposed a new theory of shock dynamics (NTSD) in 1990-91 (Prasad 1993) according to which the system of equations (2.7), (2.8), (2.11) and (2.14) can be closed by dropping the term containing $\mu_{2}$ in (2.14).

Unlike Whitham's heuristic theory of shock dynamics, the NTSD is based on only one assumption, which is dropping the last term on the right-hand side of (2.14). This step is justified in the case $\mu_{1}>0$, which occurs very frequently in applications such as a blast wave. For a single conservation law, equation (2.28) of Prasad (1993) shows that when $\phi^{\prime}(\xi)>0$, i.e. when $\mu_{1}>0$, the second derivative $u_{x x}$ at the shock, namely $\mu_{2}$, monotonically decreases as $t$ increases and for large $t, \mu_{2} \sim t^{-3}$. The numerical results presented in $\S 5$ clearly justify use of a theory with $\mu \mu_{2}$ neglected. The NTSD is not expected to give good results when $\mu_{1}<0$. In this case, (2.28) of Prasad (1993) shows that for a single conservation law in one space dimension, both $\mu_{1}$ and $\mu_{2}$ tend to infinity at a finite time. In this paper, we consider only the case $\mu_{1}>0$.

When we consider propagation of even stronger shocks in gas dynamics, the results of Lazarev, Prasad \& Singh (1995) show that neglecting the term $\mu \mu_{2}$ in the second 
compatibility condition gives good results not only when $\mu_{1}>0$ but also when $\mu_{1}$ is small but less than zero (as exemplified by the accelerating piston problem with small piston acceleration after the initial push of the piston). Thus NTSD is quite a robust method for gas dynamics. The results presented in Prasad, Ravindran \& Sau (1991) show that even when $\mu_{1}>0$, Whitham's theory may give more than $100 \%$ error. In this case, the NTSD does give a very good result but a clear mathematical proof of the bound of the error is still not available.

The final equations of the NTSD, which we use in this paper, for the propagation of a weak shock, are (2.7), (2.8), (2.11) and (2.14) with the third term on the right-hand side of (2.14) omitted.

\section{Conservation form of the equations for two-dimensional shock propagation}

We first consider a non-dimensional coordinate system which has been nondimensionalized with respect to the sound speed $a_{0}$ of the polytropic gas at rest ahead of the shock and a suitable length scale $L$ in the problem and use the same symbols $x, y$ and $t$ for the non-dimensional coordinates. Then we introduce a shock ray coordinate system $(\xi, t)$ such that $\xi=$ constant represents a shock ray (which for a shock moving into a polytropic gas at rest is orthogonal to the successive positions of the shock) and $t=$ constant represents the shock front. Note that in this section and the rest of paper, we let $M$ be the Mach number of the shock, i.e. the shock velocity divided by the constant speed of sound in the gas ahead of it:

$$
M=1+\epsilon \frac{\gamma+1}{4} \mu .
$$

Then $M \mathrm{~d} t$ represents an element of length scale along a ray. Let $G$ be the metric corresponding to the variable $\xi$, i.e. $G \mathrm{~d} \xi$ is an element of length, say $l$ along the shock front at a time $t$. We can measure $l$ from a suitable point on the wavefront, say the point where the ray $\xi=0$ meets the wavefront. Then

$$
l=\int_{0}^{\xi} G \mathrm{~d} \xi .
$$

We choose the origin of the $(x, y)$-plane to be a suitable point on the initial shock front and ray $\xi=0$ to be the one which starts from $(0,0)$ at $t=0$. We also define a quantity $N$ by

$$
N=\frac{\gamma+1}{4} \mu_{1}
$$

Let $\Theta$ be the angle that a shock ray (i.e. the normal to the shock front at time $t$ ) makes with the $x$-axis. Then the shock ray velocity is given by $M N=M(\cos \Theta, \sin \Theta)$. A general displacement $\mathbf{d} \boldsymbol{r}=(\mathrm{d} x, \mathrm{~d} y)$ in $(x, y)$-plane is given by

$$
\mathbf{d} \boldsymbol{r} \equiv(\mathrm{d} x, \mathrm{~d} y)=(\cos \Theta, \sin \Theta) M \mathrm{~d} t+(-\sin \Theta, \cos \Theta) G \mathrm{~d} \xi .
$$

In the ray coordinate system, the subscript $t$ denotes the non-dimensional time derivative along the shock ray, i.e. $\partial_{t}$ stands for $\mathrm{d} / \mathrm{d} T$ in expression (2.9) with $T$ now representing non-dimensional time. Then equations (2.7), (2.8), (2.11) and (2.14) giving the successive positions $(X(t, \xi), Y(t, \xi))$ of a weak shock and distributions of $M$ and $N$ on it, take the form

$$
X_{t}=M \cos \Theta, \quad Y_{t}=M \sin \Theta,
$$




$$
\begin{gathered}
\Theta_{t}+\frac{1}{G} M_{\xi}=0, \\
M_{t}+\frac{1}{2} \frac{1}{G}(M-1) \Theta_{\xi}+(M-1) N=0, \\
N_{t}+\frac{1}{2} \frac{1}{G} N \Theta_{\xi}+2 N^{2}=0,
\end{gathered}
$$

where we have omitted the last term on the right-hand side of (2.14). To make this a complete set, we need to add an equation for $G$. This is the equation derived from differential geometry for the evolution of the metric $G$ along the shock front. This equation, in the notation of the present paper, is

$$
G_{t}-M \Theta_{\xi}=0
$$

and follows from relations (6.51) of Prasad (1993). This equation also follows from the conservation laws (3.12) and (3.13) below.

Equations (3.5) define rays. Equations (3.6) and (3.9) are geometric conditions. The other two, namely (3.7) and (3.8), are dynamic compatibility conditions along a shock ray.

We eliminate $\Theta_{\xi}$ from (3.7)-(3.9) to get two relations from which we derive a homogeneous relation between $M_{t}, N_{t}$ and $G_{t}$ in the form

$$
2 N M_{t}+\frac{(M-1) N}{2 G M} G_{t}-(M-1) N_{t}=0 .
$$

For the weak shock under consideration, $0<M-1 \ll 1$, we can replace $(M-1) / M$ by $(M-1)$ and get an integral of (3.10) in the form $G(M-1)^{4} N^{-2}=h(\xi)$, where $h(\xi)$ can be obtained from the distribution of $G, M$ and $N$ on the initial position of the shock front. Initially, $\xi$ is chosen to be the arclength along the shock front. Therefore, initially, $G=1$. By changing $\xi$ to another function of $\xi$, (say $\xi^{\prime}$ ), it is possible to choose $h(\xi)=1$. This gives

$$
G=(M-1)^{-4} N^{2} .
$$

We denote $\xi^{\prime}$ by $\xi$.

The system of equations (3.5)-(3.8) where $G$ is given by (3.11) forms a complete set of equations to give successive positions of a shock front and distribution of $M$ and $N$. The system (3.6)-(3.8) decouples from (3.5) and forms a hyperbolic system with characteristic velocities $0, \pm \sqrt{(M-1) / 2 G^{2}}$ in the $(\xi, t)$-plane.

The characteristic fields of the non-zero eigenvalues are genuinely nonlinear and in the solutions of (3.6)-(3.8), shocks appear in the $(\xi, t)$-plane. Study of these shocks which map into kinks on the shock front in the $(x, y)$-plane, would require the conservation form of (3.6)-(3.8). From (3.4), we get expressions for $x_{\xi}, y_{\xi}, x_{t}$ and $y_{t}$. We use these to derive expressions for the second derivatives and equate $x_{\xi t}=x_{t \xi}$ and $y_{\xi t}=y_{t \xi}$. These give the following conservation laws first obtained by Morton, Prasad \& Ravindran (1992):

$$
\begin{aligned}
& (G \sin \Theta)_{t}+(M \cos \Theta)_{\xi}=0, \\
& (G \cos \Theta)_{t}-(M \sin \Theta)_{\xi}=0 .
\end{aligned}
$$

These are purely geometric results, true for any propagating curve with velocity of propagation $M$ and with the associated orthogonal family of curves as rays. For continuously differentiable functions $M$ and $\Theta$, which satisfy the above conservation laws, we can easily deduce the equations (3.6) and (3.9) from (3.12) and (3.13). 
However, as mentioned above, we also have discontinuous solutions $M$ and $\Theta$ in which the discontinuities are shocks for which the shock velocity $S$ in the $(\xi, t)$-plane satisfies the jump relations

$$
S[G \sin \Theta]-[M \cos \Theta]=0, \quad S[G \cos \Theta]+[M \sin \Theta]=0,
$$

where $[f]$ is the usual notation for the jump $f_{-}-f_{+}$of the quantity $f$ across the discontinuity. These shocks are mapped into kinks in the $(x, y)$-plane by the mapping (3.5).

We shall now show that the jump relations (3.14) imply conservation of distance in the $x$ - and $y$-directions in a sense that the vector displacement $(\mathbf{d r})_{k}$ of a kink in an infinitesimal time interval $\mathrm{d} t$ when computed in terms of the variables $\left(G_{-}, M_{-}, \Theta_{-}\right)$ and $\left(G_{+}, M_{+}, \Theta_{+}\right)$on the two sides of the kink path is the same. This result was first proved by Giles et al. (1996) in a more general context of the propagation of a three-dimensional nonlinear wavefront. In the two-dimensional case, the expression (3.4) for a displacement along a kink path gives

$$
\begin{aligned}
(\mathbf{d} \boldsymbol{r})_{k} & =\left(\cos \Theta_{-}, \sin \Theta_{-}\right) M_{-} \mathrm{d} t+\left(-\sin \Theta_{-}, \cos \Theta_{-}\right) G_{-} \mathrm{d} \xi \\
& =\left(\cos \Theta_{+}, \sin \Theta_{+}\right) M_{+} \mathrm{d} t+\left(-\sin \Theta_{+}, \cos \Theta_{+}\right) G_{+} \mathrm{d} \xi .
\end{aligned}
$$

Taking the first component of the above relation, dividing by $\mathrm{d} t$ and using $\mathrm{d} \xi / \mathrm{d} t=S$, we get the first jump relation in (3.14). Similarly the second jump relation in (3.14) also follows from (3.15). Thus, we have shown that the conservation laws (3.12) and (3.13) are physically realistic because they represent conservation of distance in the sense explained above.

For a discontinuous solution, different conservation laws with $\xi, t$ as independent variables are, in general, not equivalent. Hence (3.12) and (3.13) are the only physically realistic conservation laws.

The third conservation form is obtained from the consideration of the flow of energy along a ray tube. Eliminating $\Theta_{\xi}$ from (3.7) and (3.9), we get

$$
M_{t}+\frac{M-1}{2 G M} G_{t}+(M-1) N=0 .
$$

Since the second term has $M-1$ in the numerator, we can set $M=1$ in the denominator as explained earlier. This leads to a form

$$
\left(G(M-1)^{2}\right)_{t}+2 G(M-1)^{2} N=0 .
$$

In a small-amplitude theory, the quantity $G(M-1)^{2}$ is interpreted as the energy density along a ray tube (per unit length in $\xi$ variable). This quantity remains constant as we move with the wave along the ray in the linear theory, i.e. $\left(G(M-1)^{2}\right)_{t}=0$. In a nonlinear theory, there is an additional term due to dissipation through the shock and we take (3.17) as the third conservation law. There appears to be no other physically realistic guiding principle from which another conservation law can be deduced. Since (3.11) has been obtained as an integral (though an approximate one) of the governing equations (3.6)-(3.9), we treat it as a relation valid along a shock ray even when it crosses a kink. This is an assumption: the function $h(\xi)$ mentioned following (3.10) need not be the same on the two sides of the kink. A detailed discussion of this point is available in Whitham (1974). Thus we take the fourth conservation law as

$$
\left(G(M-1)^{4} N^{-2}\right)_{t}=0 .
$$

The equations (3.12), (3.13), (3.17) and (3.18) form the system of conservation laws 
which with appropriate initial values can be solved numerically in $(\xi, t)$-plane. By numerically integrating the two equations (3.5), the solution can be mapped onto the $(x, y)$-plane giving the successive positions of the shock front and the distribution of amplitude on them.

Experimental results of Sturtevant \& Kulkarni (1976) show that in the linear case a complex wave field develops near the focus of the wavefront. If the shock wave is relatively weak, the wavefront emerges from focus crossed and folded (in accordance with the predictions of geometric acoustics theory), whereas, in the stronger shock case, the caustic of the linear theory is resolved and the shock front beyond the focus is uncrossed, as predicted by the theory of shock dynamics. In both cases, the behaviour at the focus is nonlinear. Explanation or determination of the transition from the linear to fully nonlinear behaviour (of uncrossed geometry with kinks) is a very important and challenging mathematical problem. It appears that the present mathematical formulation may not be able to describe this transition because as $M \rightarrow 1, G$ could tend to infinity according to (3.11) or equivalently, (3.18).

We present the results of extensive numerical solutions of the conservative form of four equations for quite general forms of initial data for $M$ and $N$ and also $X$ and $Y$ giving initial position of shock. We are able to numerically simulate the experimentally observed behaviour when the caustic is resolved. A total-variationbounded (TVB) scheme based on the Lax-Friedrichs flux has been used to solve this system of conservation laws. The source term in the equation was handled through Strang splitting (LeVeque \& Yee 1990). The effects of varying initial curvature and the effects of varying the initial Mach strength $M$ and normal derivative $N$ on the formation and propagation of kinks has been established.

\section{Initial conditions and description of numerical method}

We consider an initially concave shock front moving from left to right (i.e. in the positive direction of the $x$-axis into a gas at rest).

Let

$$
X(\xi, 0)=X_{0}(\xi), \quad Y(\xi, 0)=Y_{0}(\xi)
$$

denote the initial shock front with an initial distribution of amplitude $M$ and $N$ given by

$$
\begin{aligned}
M(\xi, 0) & =M_{0}(\xi), \\
N(\xi, 0) & =N_{0}(\xi) .
\end{aligned}
$$

The initial value of $\Theta$ is obtained from (4.1) as

$$
\Theta(\xi, 0)=\Theta_{0}(\xi)
$$

and the initial value of $G$ is obtained from the relation

$$
G=(M-1)^{-4} N^{2}
$$

as

$$
G(\xi, 0)=G\left(M_{0}(\xi), N_{0}(\xi)\right)=G_{0}(\xi) .
$$

\subsection{A finite difference scheme}

To solve the initial value problem (3.12), (3.13), (3.17) and (3.18) with (4.1)-(4.6), we use a total-variation-bounded finite difference scheme based on the Lax-Friedrichs flux (Shu 1987; Cockburn, Lin \& Shu 1989). Application of the scheme to the system 
of conservation laws (3.12), (3.13), (3.17) and (3.18) is based on the decomposition of these equations into characteristic components. The scheme is then applied to individual characteristic components and the solution is obtained from summation of the contributions from the characteristic components. Consider the conservation law

$$
\boldsymbol{u}_{t}+(\boldsymbol{f}(\boldsymbol{u}))_{x}=0
$$

where $\boldsymbol{u}=\left(u_{1}, \ldots, u_{m}\right)^{t}$ and the Jacobian matrix $\boldsymbol{A}(\boldsymbol{u})=\partial \mathbf{f} / \partial \boldsymbol{u}$ has $m$ real eigenvalues and its eigenspace is complete (i.e. (4.7) is hyperbolic).

On the computational grid $x_{j}=j \Delta x, t_{n}=n \Delta t$, and $\boldsymbol{u}_{j}^{n}$ denotes the computed approximation to the exact solution $\boldsymbol{u}\left(x_{j}, t_{n}\right)$ of (4.7).

Let $\boldsymbol{A}_{j+1 / 2}=(\partial \boldsymbol{f} / \partial \boldsymbol{u})_{\boldsymbol{u}=\boldsymbol{u}_{j+1 / 2}}$ denote the average Jacobian where

$$
\boldsymbol{u}_{j+1 / 2}=\left(\frac{\boldsymbol{u}_{j}^{(0)}+\boldsymbol{u}_{j+1}^{(0)}}{2}\right) .
$$

We denote the eigenvalues and left and right eigenvectors of $\boldsymbol{A}_{j+1 / 2}$ by

$$
\lambda_{j+1 / 2}^{(p)}, \quad \boldsymbol{l}_{j+1 / 2}^{(p)}, \quad \boldsymbol{r}_{j+1 / 2}^{(p)}, \quad p=1,2,3,4,
$$

normalized so that

$$
\boldsymbol{l}_{j+1 / 2}^{(p)} \cdot \boldsymbol{r}_{j+1 / 2}^{(q)}=\delta_{p q}
$$

Denote

$$
\Delta_{+} \boldsymbol{u}_{j}=\boldsymbol{u}_{j+1}-\boldsymbol{u}_{j}, \quad \Delta_{-} \boldsymbol{u}_{j}=\boldsymbol{u}_{j}-\boldsymbol{u}_{j-1} .
$$

A semidiscrete method of lines to (4.7) is a system of ordinary differential equations

$$
\frac{\partial \boldsymbol{u}_{j}}{\partial t}=-\frac{1}{\Delta x}\left(\boldsymbol{F}_{j+1 / 2}-\boldsymbol{F}_{j-1 / 2}\right)
$$

where the numerical flux $\boldsymbol{F}_{j+1 / 2}$ is defined by

$$
\boldsymbol{F}_{j+1 / 2}=\boldsymbol{F}\left(u_{j-k+1}, \ldots, u_{j+k}\right)
$$

for some positive integer $k . \boldsymbol{F}_{j+1 / 2}$ is Lipschitz continuous in all its arguments and satisfies the consistency condition

$$
F(u, \ldots, u)=f(u) .
$$

Taking the Euler forward time discretization of (4.11), we get

$$
\boldsymbol{u}_{j}^{n+1}=\boldsymbol{u}_{j}^{n}-\lambda\left(\boldsymbol{F}_{j+1 / 2}^{n}-\boldsymbol{F}_{j-1 / 2}^{n}\right)
$$

where $\lambda=\Delta t / \Delta x$ is the CFL number. In the TVB modification, the numerical flux $\boldsymbol{F}_{j+1 / 2}$ is replaced by a monotone flux (whose choice is defined later)

$$
\boldsymbol{h}_{j+1 / 2}=\boldsymbol{h}\left(\boldsymbol{u}_{j+1 / 2}^{-}, \boldsymbol{u}_{j+1 / 2}^{+}\right)
$$

where $\boldsymbol{u}_{j+1 / 2}^{ \pm}=\boldsymbol{u}\left(x_{j+1 / 2}^{ \pm}, t\right)$ are defined suitably subject to some local projection limiting.

First, project everything to the eigenspace of $\boldsymbol{A}_{j+1 / 2}$,

$$
a^{(p)}=\boldsymbol{l}_{j+1 / 2}^{(p)} \cdot \boldsymbol{a}
$$

where we take $\boldsymbol{a}=\boldsymbol{u}_{j}^{(0)}, \boldsymbol{u}_{j+1}^{(0)}, \Delta_{-} \boldsymbol{u}_{j}^{(0)}, \Delta_{+} \boldsymbol{u}_{j}^{(0)}, \Delta_{-} \boldsymbol{u}_{j+1}^{(0)}$. 
Then apply the local projection limiting in each characteristic field:

$$
\begin{gathered}
\left(\tilde{u}_{j}\right)^{(p)}=m\left(\left(u_{j}^{(0)}\right)^{(p)},\left(\Delta_{+} u_{j}^{(0)}\right)^{(p)},\left(\Delta_{-} u_{j}^{(0)}\right)^{(p)}\right), \\
\left(\tilde{u}_{j+1}\right)^{(p)}=m\left(\left(u_{j+1}^{(0)}\right)^{(p)},\left(\Delta_{+} u_{j}^{(0)}\right)^{(p)},\left(\Delta_{+} u_{j+1}^{(0)}\right)^{(p)}\right),
\end{gathered}
$$

where $m$ is the minmod function, i.e.

$$
m\left(b_{1}, b_{2}, \ldots, b_{n}\right)= \begin{cases}\Delta \min _{1 \leqslant i \leqslant n}\left|b_{i}\right|, & \text { if } \operatorname{sgn}\left(b_{1}\right)=\operatorname{sgn}\left(b_{2}\right)=\cdots=\operatorname{sgn}\left(b_{n}\right)=\Delta \\ 0, & \text { otherwise. }\end{cases}
$$

We then form

$$
\begin{gathered}
\left(u_{j+1 / 2}^{-}\right)^{(p)}=\left(u_{j}^{(0)}\right)^{(p)}+\Phi\left(\tilde{u}_{j}\right)^{(p)}, \\
\left(u_{j+1 / 2}^{+}\right)^{(p)}=\left(u_{j+1}^{(0)}\right)^{(p)}-\Phi\left(\tilde{u}_{j+1}\right)^{(p)},
\end{gathered}
$$

where $\Phi$ is the limiter, $0<\Phi<1$. Also, $\Phi=0$ gives the Lax-Friedrichs flux.

Taking $a=u_{j+1 / 2}^{ \pm}$, we return to the component space using

$$
\boldsymbol{a}=\sum_{p=1}^{m} a^{(p)} \cdot r_{j+1 / 2}^{(p)}
$$

and find $\boldsymbol{f}_{j+1 / 2}^{ \pm}=\boldsymbol{f}\left(\boldsymbol{u}_{j+1 / 2}^{ \pm}\right)$.

Next, we compute $\left(f_{j+1 / 2}^{ \pm}\right)^{(p)}$ from (4.16), and then use any scalar monotone flux in the $p$ th characteristic field, $p=1,2, \ldots, m$. We have used the local Lax-Friedrichs flux defined by

$$
\begin{aligned}
h_{j+1 / 2}^{(p)} & =h\left(\left(u_{j+1 / 2}^{-}\right)^{(p)},\left(u_{j+1 / 2}^{+}\right)^{(p)}\right) \\
& =\frac{1}{2}\left[\left(f_{j+1 / 2}^{-}\right)^{(p)}+\left(f_{j+1 / 2}^{+}\right)^{(p)}-\mathscr{L}_{j+1 / 2}^{(p)}\left\{\left(u_{j+1 / 2}^{+}\right)^{(p)}-\left(u_{j+1 / 2}^{-}\right)^{(p)}\right\}\right]
\end{aligned}
$$

with

$$
\mathscr{L}_{j+1 / 2}^{(p)}=\max \left(\left|\lambda_{j}^{(p)}\right|,\left|\lambda_{j+1}^{(p)}\right|\right) .
$$

We finally obtain $\boldsymbol{h}_{j+1 / 2}$ by making use of (4.22) with $a=h_{j+1 / 2}$

$$
\boldsymbol{h}_{j+1 / 2}=\sum_{p=1}^{m} h_{j+1 / 2}^{(p)} \cdot r_{j+1 / 2}^{(p)}
$$

Now, replacing $\boldsymbol{F}_{j+1 / 2}$ by $\boldsymbol{h}_{j+1 / 2}$ in (4.14), the TVB finite difference scheme can be written as

$$
\boldsymbol{u}_{j}^{n+1}=\boldsymbol{u}_{j}^{n}-\lambda\left(\boldsymbol{h}_{j+1 / 2}^{n}-\boldsymbol{h}_{j-1 / 2}^{n}\right) .
$$

The above scheme can be applied to the system of equations (3.12), (3.13), (3.17) and (3.18). The source term in equation (3.16) is handled by employing a time splitting in which one alternates between solving a system of conservation laws with no source terms, and a system of ordinary differential equations. In the simplest case, this splitting takes the form (LeVeque \& Yee 1990)

$$
u^{n+1}=S_{\Psi}(k) S_{f}(k) u^{n}
$$

where $S_{f}(k)$ represents the numerical solution operator for the system of conservation laws

$$
\boldsymbol{u}_{t}+(\boldsymbol{f}(\boldsymbol{u}))_{x}=0
$$


over a time step of length $k$, and $S_{\Psi}(k)$ is the numerical solution operator for the ODE system

$$
\boldsymbol{u}_{t}=\Psi(\boldsymbol{u})
$$

To maintain second-order accuracy, we use the Strang splitting in which the solution $U^{n+1}$ is computed from $U^{n}$ by (Strang, 1968)

$$
U^{n+1}=S_{\Psi}(k / 2) S_{f}(k) S_{\Psi}(k / 2) U^{n} .
$$

The fact that the splitting is second-order accurate suggests that the interaction of different effects is adequately modelled by a split method (at least for smooth solutions). Moreover, there are distinct advantages to using the splitting form from the standpoint of algorithm design. High-quality numerical methods have been developed for systems of conservation laws. By decomposing the problem into subproblems of these types, it is possible to take advantage of these methods directly.

To assess the accuracy, we first applied the method to a Burgers equation with source term

with initial condition

$$
u_{t}+\left(\frac{1}{2} u^{2}\right)_{x}=k u, \quad k=\mathrm{constant}
$$

$$
u(x, 0)= \begin{cases}x, & 0<x<1 \\ 0, & \text { elsewhere }\end{cases}
$$

An exact solution of this problem can easily be obtained. We find that the solution obtained numerically agrees with the exact solution up to four significant figures in the shock-free region when $\Delta x=0.01, \Delta t=0.01$ and cannot be distinguished on the graph from the solution obtained analytically. Thus the numerical method is accurate for this test case.

\subsection{Method of construction of the shock front and the rays}

The governing equations (3.12), (3.13), (3.17) and (3.18) are discretized on a twodimensional grid along the $\xi$-direction and $t$-direction in the $(\xi, t)$-plane. The solution of the discrete equations is obtained starting with initial data for different sets of values $\Theta_{0}, M_{0}, N_{0}$ and $G_{0}$. The numerical procedure for obtaining the successive positions of a shock front and rays is as follows.

Along a ray given by $\xi=\xi_{0}, M$ and $\Theta$ are known from the solution of the above discretized system. Integrating the equations

$$
X_{t}=M \cos \Theta, \quad Y_{t}=M \sin \Theta
$$

along $\xi=\xi_{0}$, with initial values given by (4.1), we can find $(X, Y)$ at any time $t>0$ on the shock front. The composite Simpson's rule was used for integration. We store these data and then vary $\xi_{0}$. This procedure gives both the rays (joining points $(X, Y)$ on $\xi_{0}=$ constant for various values of $t$ ) and the shock front (joining points $(X, Y)$ for various values of $\xi_{0}$ at a given $t$ ).

\section{Results and discussion}

In this section, we discuss the geometrical features of the successive positions of a shock front and the amplitude distribution on it as obtained from computations which have been carried out by varying the distribution of amplitude along different initial shock fronts. To save space we do not reproduce here many of the figures from which conclusions have been derived (see Monica 1999, for these figures). The following cases are considered. 
5.1. Case (i): Propagation of a shock front initially parabolic in shape

The initial shock front is taken as

$$
y^{2}=b x, \quad 1 \leqslant b \leqslant 8, \quad|y| \leqslant z,
$$

extended on either side as a straight line for $|y|>z . M_{0}$ is prescribed on the initial front as a symmetric function of $\Theta_{0}$. We take

$$
M_{0}=\alpha \exp \left(-\beta \Theta_{0}^{2}\right)
$$

where the parameter $\alpha$ is a measure of the strength of the initial shock front and $\beta$ is a measure of the rate of change of $M_{0}$ along the shock front. The distribution of amplitude on the shock front can be varied by varying $\alpha$ and $\beta$.

We also prescribe the initial value of $N$. For most cases, we have taken $N_{0}$ to be a constant and have varied it from 0.1 to 1.0 .

\subsubsection{Effect of varying the initial amplitude distribution}

Computations were carried out for $b=2,4,8$. For each value of $b$, the initial distribution of $\alpha$ was varied between 1.1 and 1.2. We present below some of the representative cases. We start with general qualitative behaviour from one case.

Figure 2 with $b=8, z=1$ is typical of the results of computation, part (a) shows the successive positions of the shock front and $(b-e)$ the distributions of $M, \Theta, N$ and $G$ on the shock at different times marked by the values of $t$ from $t=0$ to 10 at time interval two. The shock rays, except the central one, initially tend to converge but later deviate from a straight path due to the non-uniform distribution of the amplitude (according to equation (3.6)) and become parallel to the $x$-axis. Thus the formation of the caustic (which happens in the linear theory) is avoided and the propagating shock front becomes plane in the central region. A pair of kinks, shown in figure 2(a) by dots, appears on the shock. The position of the kink on the shock is very accurately determined by the sharp gradient from the $M, l$ graph in figure $2(b)$. The ultimate shape of the shock front consists of a plane central disc separated from straight wings on the two sides by the two kinks. In this and all the cases we considered in our numerical computation, we found that the kinks move away from one another leaving an ever increasing shock disc. There is, though, a definite condition (Kevlahan 1994; Prasad 2001) involving the values of $M$ on the two sides of the kink and the jump in $\Theta$ across it, which if satisfied, would make the shock disc shrink. As we shall see at the end of $\S 6.3$, both $M-1$ and $N$ tend to zero on the wings as well as on the disc as $t \rightarrow \infty$.

The strength $M_{0}$ on the shock at $t=0$ is prescribed to be constant in figure 2 . Due to convergence of rays, the shock strength in the central part increases initially but it decreases on those rays which start from the straight part (i.e. $|y|>1$ ) of the initial shock front. At a critical time $t_{c}$, depending on the values of $\alpha$ and $\beta$, the shock strength $M$ attains a maximum value on the central ray and after that it continuously decreases along all rays. The $\Theta, l$ graph in figure $2(c)$ shows that till time $t=10, \Theta$ is not constant on the disc, implying that the central disc is not plane, but it tends to a plane as time increases. The value of $N$, the gradient of the gas density (or velocity) just behind the shock, has also been prescribed to be a constant equal to 0.15 . Since the nonlinear waves from behind catch up with the shock and then disappear from the flow field, $N$ decreases continuously but with different rates along different rays. The $N, l$ graph in figure $2(d)$ shows that from $N=0.15$ at $t=0$, the value of $N$ has decreased to less than 0.06 at $t=10$. It is important to note that the shape of the shock front is qualitatively the same as that of the nonlinear wavefront (Prasad \& 
(a)

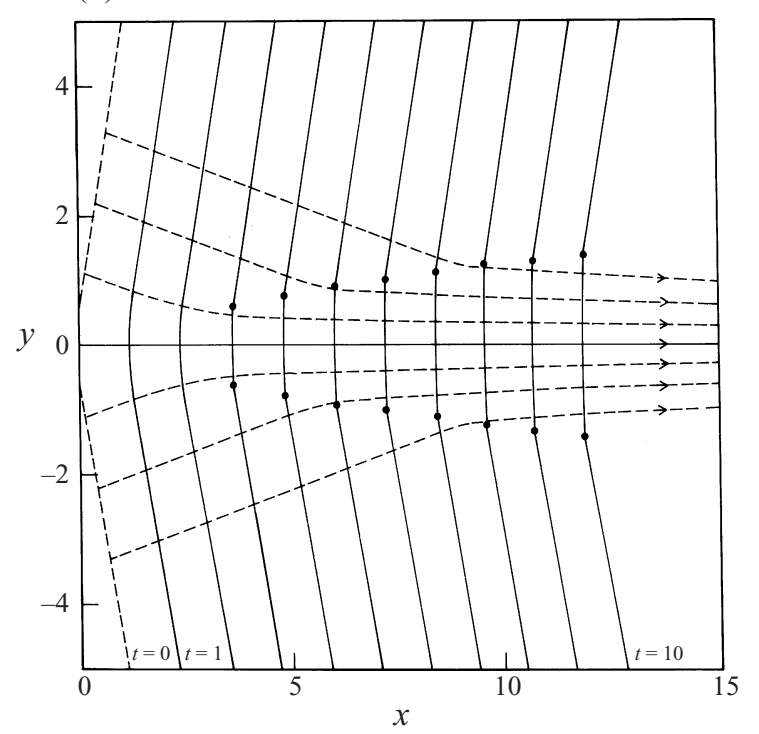

(b)

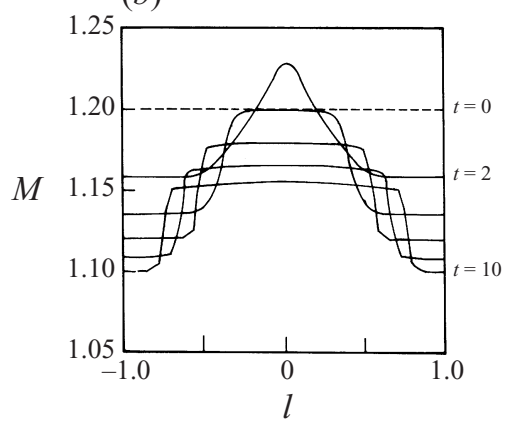

(d)

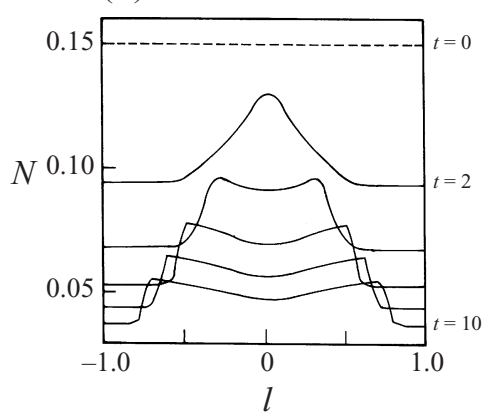

(c)

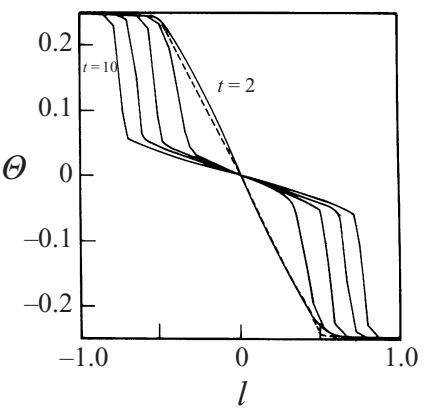

(e)

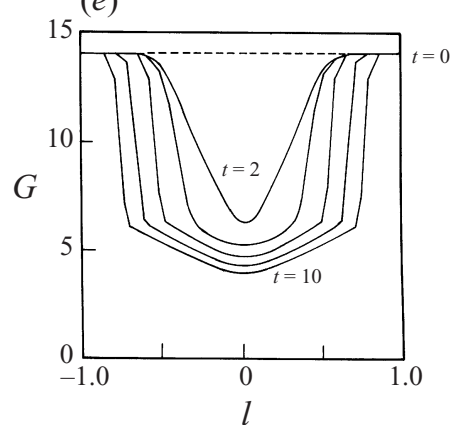

FIGURE 2. Propagation of a weak nonlinear shock front starting from an initial shock front wherein the central part is a parabola $y^{2}=8 x$ for $|y|<1$. The initial amplitude distribution is given by $M_{0}=1.2$ and $N_{0}=0.15$. The dotted curves are the rays. (a) Successive positions of the shock front. $(b-e)$ Distributions of $M, \Theta, N$ and $G$. 
Sangeeta 1999). Figure 2(e) shows that the metric $G$, which was constant initially with value about 14, decreases significantly in the central part of the shock front. For an efficient numerical computation, the lower bound of $G$ should be greater than zero.

We have done a number of computations changing the value of $M_{0}$ all over the initial front by changing the value of $\alpha$ (say 1.15, 1.12,1.10) and keeping all the other parameters in the initial data of the case represented in figure 2 the same. We found that as $\alpha$ increases

$N$ decreases faster along each ray,

the shock disc becomes larger, and

the shock front moves faster-an obvious result.

It was also found that the maximum value $M$, which is attained at $t=t_{c}$ on the central ray, always appears before the kinks appear. Next, the initial value of $M_{0}$ is so prescribed that it is maximum (minimum) at the centre and decreases (increases) monotonically to the values on the two sides. These values of $M_{0}$ are chosen in such a way that they coincide on the straight line part, but are different on the parabolic part of the initial front. In figure 3 , we have chosen an initial shock front with the values $b=8, z=1$ and taken $\alpha=1.2, \beta=0.5$ for $M_{0}$ maximum at the centre (say case I) and $\alpha=1.1301, \beta=-0.5$ for $M_{0}$ minimum at the centre (say case II). In both cases, the $M_{0}$ on the straight line part is 1.1645 . Figure $3(c, d)$ show the comparison of the graph of $M$ in the two cases. The solid lines correspond to case I and the dotted lines correspond to case II. Although the maximum value of $M$ at a given time in case I is more than the corresponding value in case II before the critical time (i.e. the time at which the shock front develops kinks), the behaviour is reversed after the critical time. We note that the smaller value of $M_{0}$ in the central region leads to a delay in the formation of kinks and to a slight lagging of the central disc initially. However, as time increases, the constant value on the wings ultimately determines the geometry of the shock front and the amplitude distribution. This is expected as the value of $M_{0}$ is changed only on a small bounded interval near the origin on the $\xi$-axis leaving the value of $M_{0}$ unchanged outside this interval. The distribution of $M_{0}$ on this outside infinite part determines the ultimate shape and the shock strength of the shock front.

An interesting result to note from figure $3(c, d)$ is that as $M_{0}$ is decreased, the central part of the shock front remains more concave for a longer time, leading to the convergence of the rays for a longer time which ultimately pushes the value of $M$ to a larger value. This result was also observed in the WNLRT (Prasad \& Sangeeta 1999).

\subsubsection{Effect of varying the initial $N$}

The value of $N$ represents the gradient in the normal direction of the gas density just behind the shock and hence is a measure of the rate of interaction of the nonlinear waves with the shock. As we have noted earlier, and as will be seen later, this interaction, for $N>0$, leads to the ultimate decay of the shock strength to zero. Larger value of $N$ means a higher rate of interaction leading to faster decay of the shock. In all calculations in this paper, we have taken $N>0$, i.e. expansion waves behind the shock. Taking $N<0$ initially may lead to a very large and even infinite value of the shock strength $M-1$ showing that the small-amplitude assumption breaks down and this theory is no longer valid. In figure 4, we present results of computation with $N_{0}=0.15$ and 0.5 , keeping all the other parameters same. We note that when $N_{0}$ is increased everywhere on the initial shock front (and not just on the curved part) 
(a)

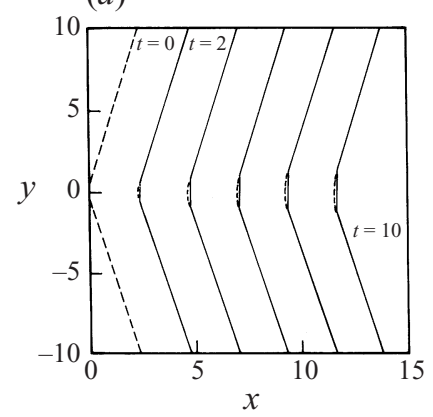

(c)

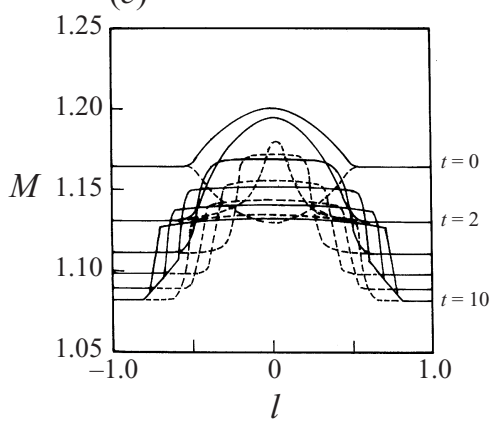

(b)

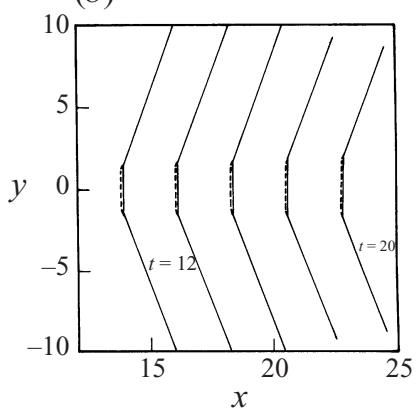

(d)

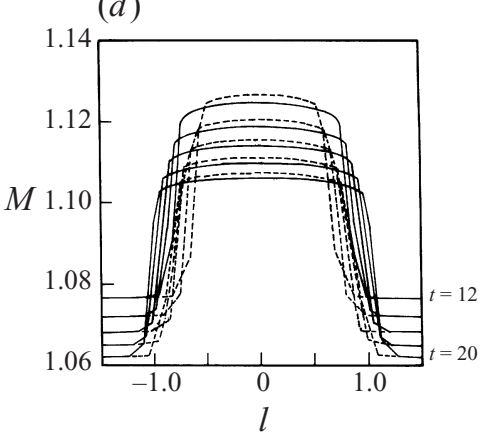

FIGURE 3. Comparison of the results when the amplitude distribution is decreased on the parabolic part of the initial shock front $(b=8, z=1)$. Solid lines: results for $\alpha=1.2, \beta=0.5$; dotted lines: results for $\alpha=1.1301, \beta=-0.5$.

shock strength decreases everywhere on the shock front faster as $t$ increases (see Monica 1999 for figures showing this result),

the shock position lags behind,

the shock disc becomes smaller,

the kinks are formed later, and

$M_{\max }(t)-M_{\min }(t)$ initially increases and then decreases with time,

where we define

$$
M_{\max }(t)=\max _{\xi \in \mathbb{R}} M(\xi, t), \quad M_{\min }(t)=\min _{\xi \in \mathbb{R}} M(\xi, t) .
$$

\subsubsection{Effect of varying geometry of the initial shock front}

We first consider the effect of changing $z$ in (5.1), so that the inclination to the $x$-axis of the wings of the initial shock front changes without changing the curvature at the centre. When $z$ in (5.1) is increased from $z=1$ to $z=2$, the central curved part of the front is increased and the inclination of the wings with respect to the $x$-axis is decreased but the curvature at the centre of the front does not change with $z$. A comparison of the results shows that when $z$ is increased

the rays from the wings converge more strongly leading to a stronger shock strength in the centre,

the shock disc between the two kinks becomes smaller, and

the value of $N$ decreases more slowly showing that the interaction of the waves from behind is slower. 

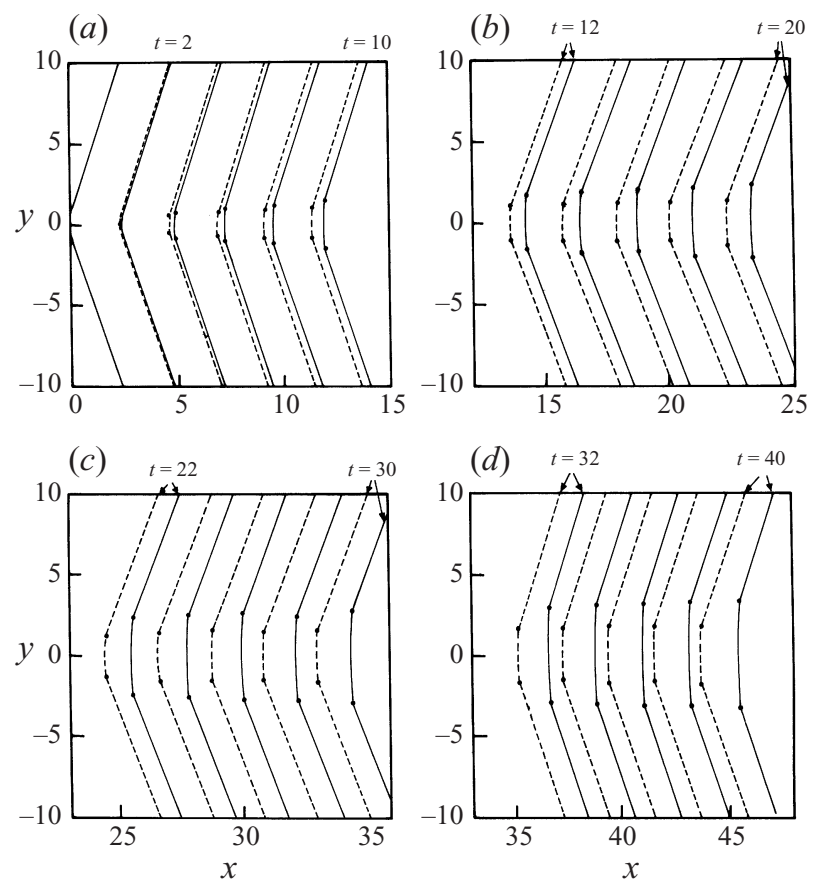

FIGURE 4. It shows the effect of increase in the value of $N_{0}$, on the shock position. Initial shock front is for $b=8, z=1$ with $\alpha=1.2, \beta=0$. Solid lines: $N_{0}=0.15$; dotted lines: $N_{0}=0.5$.

Next, we increase the curvature of the initial shock front at the centre by increasing the value of $b$. This leads to

a higher value of the shock strength $M-1$ and the normal derivative $N$ near the centre, and

a smaller shock disc.

However, as time increases, $M-1$ and $N$ on the disc seem to approach the same value as that for the case $z=1$. This is to be expected as the long-time behaviour of the solution is determined by the initial value of the shock strength on the wings.

\subsubsection{Effect of increasing the shock strength on the initial shock front}

Increase of $\alpha$ leads to an obvious result of the shock moving faster. It also resulted in an increase in the distance separating the two kinks (see also Prasad \& Sangeeta 1999, figure 9).

\subsection{Case (ii): propagation of a shock front with initially sinusoidal shape and} periodic amplitude distribution

We consider now the initial shock front to be of a periodic sinusoidal shape

$$
x=0.2-0.2 A \cos (\pi y / B), \quad \text { where } A=1, B=2,
$$

where $M_{0}$ is prescribed as in (5.2) with $\alpha=1.2$ and $\beta=0$ and $N_{0}=0.1$. Figure 5 shows the shock fronts and the associated rays at successive times. The convergence and divergence of the rays is seen quite clearly in figure $5(a)$, i.e. during the time interval $(0,10)$. With increasing time, the rays become straight lines parallel to the $x$-axis and the shock front tends to become planar.

A pair of kinks appears in each period of the shock front at $t \approx 2$. Two kinks 
(a) $t=0$ to 10

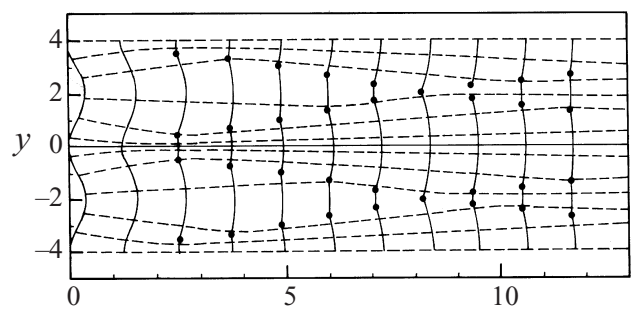

(b) $t=21$ to 30

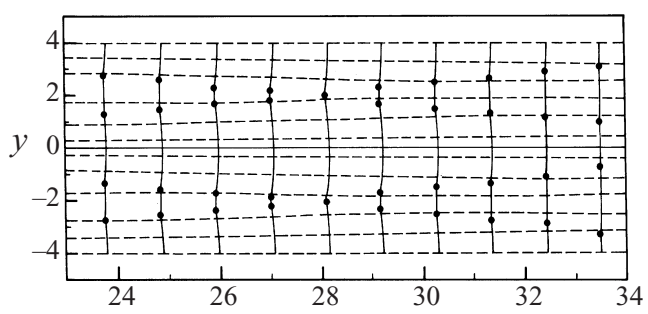

(c) $t=31$ to 40

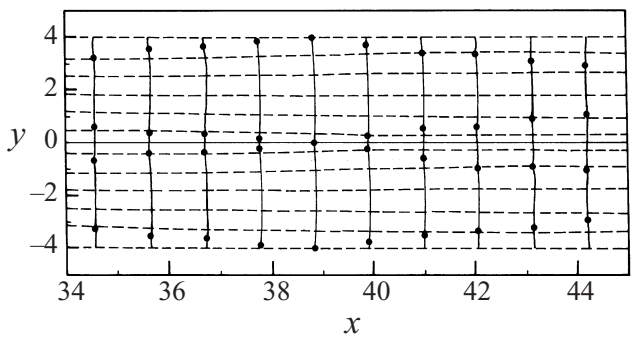

FIGURE 5. Successive positions of an initially sinusoidal shock front and rays plotted at $t=0,1,2,3, \ldots, 40$. Initial shock front is $x=0.2-0.2 \cos (\pi y / 2)$ with $M_{0}=1.2$ and $N_{0}=0.1$.

suitably paired first move closer to one another, and then interact, producing a new pair of kinks which move apart, and the process continues. After a long period of time, the amplitude $M-1$ decays to zero along each ray. The total variation of $M$ in each period keeps on decreasing with increasing time and $M-1 \rightarrow 0$ as $t \rightarrow \infty$. After a long time, even when the shock front has become almost a straight line, it is not difficult to locate the position of the kinks. At any time $t$, the kinks are easily located from the $(M, l)$ graph as shocks (see figure 6).

As before, we define $M_{\max }(t)\left(M_{\min }(t)\right)$ as the maximum (minimum) Mach number attained on the front at a given time $t . M_{\max }(t)$ attains a maximum value greater than $M_{\max }(0)$ at $t=2$, which is approximately the time when the kinks first appear. Similarly, $M_{\min }(t)$ attains a minimum value at $t=6$, the time when the kinks first interact. Figure 7 shows that $M_{\max }(t)$ and $M_{\min }(t)$ both tend to 1 and $M_{\max }(t)-$ $M_{\text {min }}(t) \rightarrow 0$ as $t \rightarrow \infty$. We note that when the kinks interact, $M_{\max }(t)$ and $M_{\text {min }}(t)$ suddenly jump from smaller to larger values - the amount of the jump tends to zero as $t \rightarrow \infty$.

The two kinks which approach each other and interact correspond to shocks belonging to two different characteristic fields with characteristic velocities $\pm \sqrt{(M-1) / 2 G^{2}}$. The shock speed in the $(\xi, t)$-plane is given by $\pm\left\{\left[M^{2}\right] /\left[G^{2}\right]\right\}^{1 / 2}$ (see Prasad 1993). 
(a)

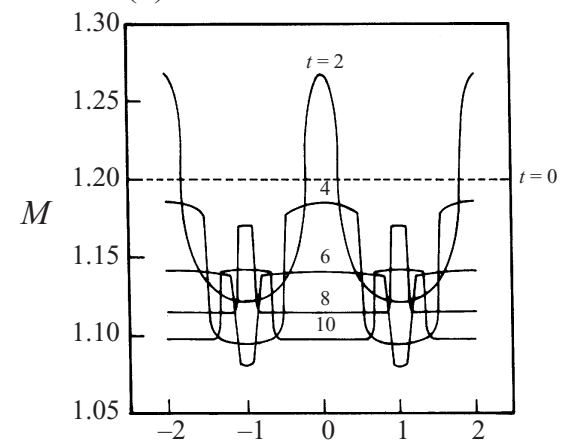

(c)

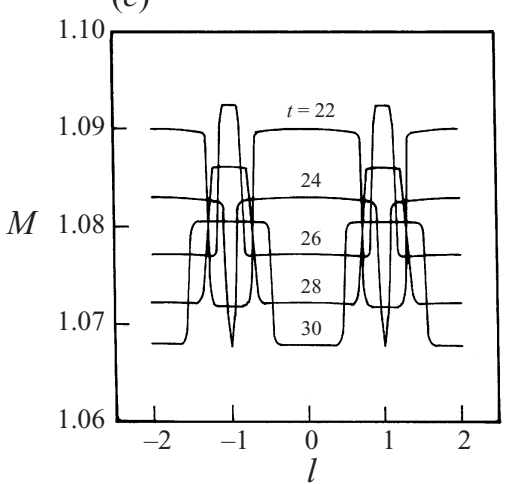

(b)

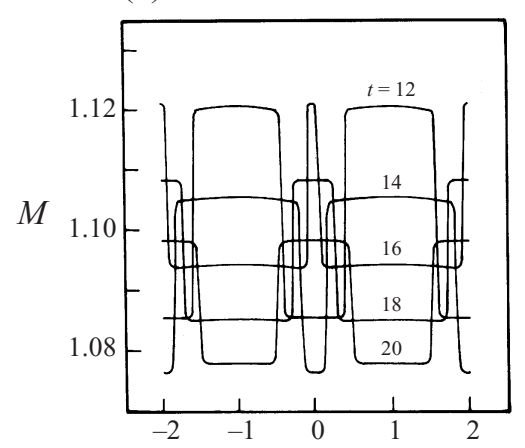

(d)

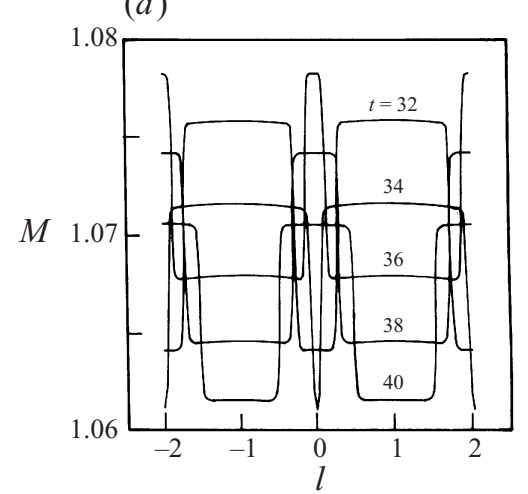

FIGURE 6. Variation of Mach number with respect to $l$ in the initially sinusoidal shock (various graphs have different scales of $M$ ). $M$ changes behaviour after the kinks interact.
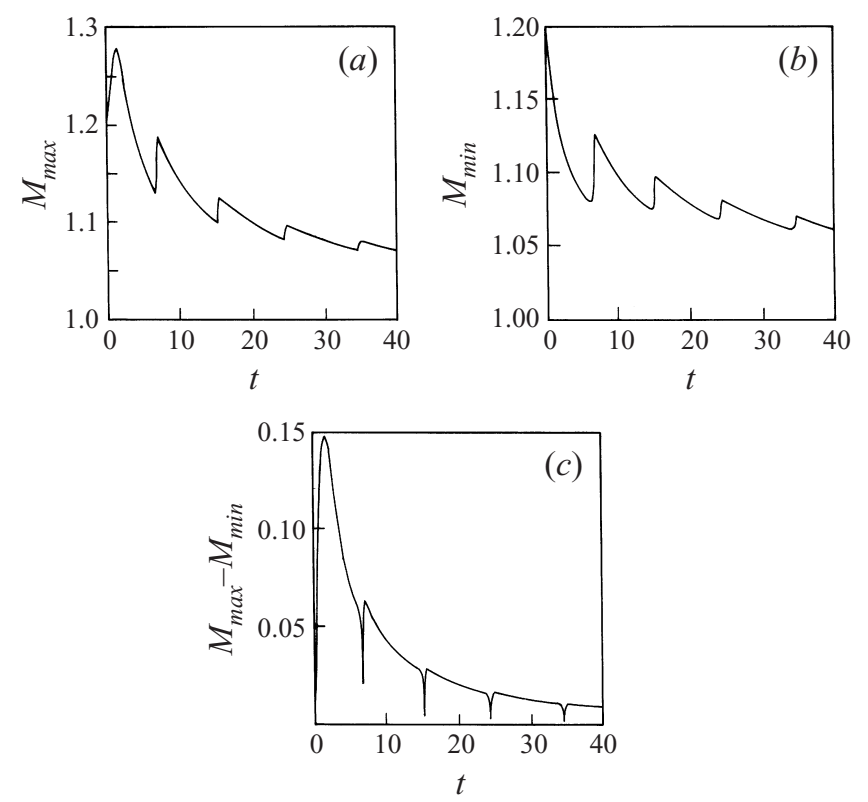

FigURE 7. Graphs of $M_{\max }, M_{\min },\left(M_{\max }-M_{\min }\right)$ with time when the initial shock front is sinusoidal. 
When these shocks interact, a new pair of shocks is produced, which leaves behind it a much higher value of $M$ as seen in figure 6 .

5.3. Case (iii): propagation of a shock front with initially asymmetric but piecewise parabolic shape in each period

The initial shock front is taken as

$$
x= \begin{cases}0.6-\frac{1}{8}(y+6.4)^{2}, & -6.4<y<-6.0 \\ -\frac{1}{10}(y+0.2), & -6.0<y<-0.4 \\ \frac{1}{8} y^{2}, & -0.4<y<1.0 \\ \frac{1}{4}(y-0.5), & 1.0<y<2.4 \\ 0.6-\frac{1}{8}(y-3.4)^{2}, & 2.4<y<3.4\end{cases}
$$

and this configuration is repeated periodically in $y . M_{0}$ has been prescribed as 1.1 .

Here too, the behaviour of the shock front is similar to the sinusoidal case except that the shape remains asymmetric in each period and with increasing time the rays become parallel to the $x$-axis and the shock front tends to become planar. This case is shown in figure 8. We note that the shock front in case (ii) has become almost planar at $t=30$ but this is not so in this case. It is not clear whether it is due to the fact that the initial shock front has a bigger oscillation in its shape or due to the lack of symmetry in its shape in each period.

5.4. Case (iv): propagation of a shock front with initially periodic but arbitrary shape in each period

An arbitrary periodic shape is not possible numerically but we choose here a shape so far removed from the symmetric shape that it may be considered practically as a representative of any unsymmetric shape. The initial shock front is formed in each period by joining a series of parts of parabolas and straight lines, the slope being continuous at the points where they are joined. This curve is given by

$$
x= \begin{cases}\frac{1}{4}(y+4.4)^{2}, & -5.0<y<-3.4 \\ \frac{1}{2}(y+3.9), & -3.4<y<-3.2 \\ -\frac{1}{4}(y+2.2)^{2}+0.6, & -3.2<y<-1.2 \\ -\frac{1}{2}(y+0.5), & -1.2<y<-1.0 \\ \frac{1}{4} y^{2}, & -1.0<y<0.4 \\ \frac{1}{5}(y-0.2), & 0.4<y<2.0 \\ -\frac{1}{4}(y-2.4)^{2}+0.4, & 2.0<y<2.8 \\ -\frac{1}{5}(y-4.6), & 2.8<y<4.4 \\ \frac{1}{4}(y-4.8)^{2}, & 4.4<y<5.0\end{cases}
$$

in $|y| \leqslant 5$ and extended for all $y$ periodically. The results of the computation for this case are shown in figure 9.

We shall make in $\S 6.5$ one observation from all computations for shock fronts of periodic shape. This concerns the corrugational stability of shocks of arbitrary shape (Anile \& Russo 1986). It implies that a shock, which is obtained by small perturbation in the shape from a plane shock, tends to become planar. 
(a) $t=0$ to 10

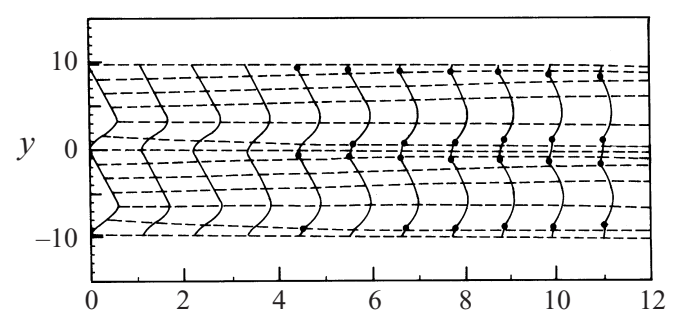

(b) $t=21$ to 30

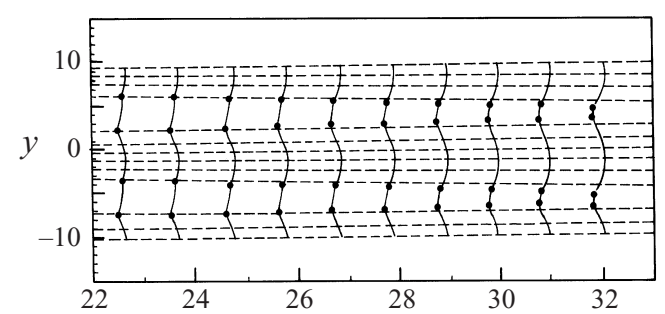

(c) $t=31$ to 40

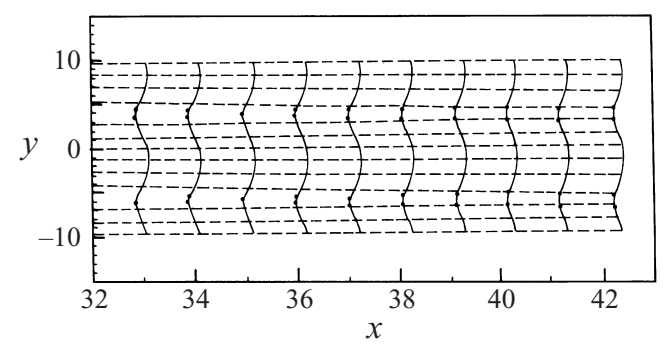

FIGURE 8. Successive positions of the shock and associated rays starting from a periodic shock, asymmetric and with piecewise parabolic shape in each period.

5.5. Case (v): when the initial shock front has a single smooth dent or bulge We take the initial shock front to be given by

$$
x= \pm \exp \left(-\frac{1}{2} y^{2}\right)
$$

where + represents a bulge and - represents a dent. The initial Mach number is prescribed as

$$
M_{0}=\alpha \exp \left(-\beta x^{2}\right)
$$

which tends to $\alpha$ as $x \rightarrow 0$ for $y \rightarrow \pm \infty$. The value of $N$ is taken to be a constant equal to 0.1 .

We first discuss the results when there is a dent. Figures 10 and 11 give the successive positions of the shock front for some representative cases. In figure $10(a)$, initially the shock Mach number at the centre is minimum, in figure $10(b)$ it is maximum and figure $10(c)$ it has the same value everywhere on the front. The graphs representing the distribution of $M, N$ and $\Theta$ with $l$ at various times can be found in Monica (1999). The amplitude first increases in the initially dented region, the increase being maximum at the centre. Elsewhere, it continues to decrease but it decreases more rapidly near the outer edges of the dent (i.e. near $l= \pm 1.25$ ). A pair of kinks is formed which moves away from the centre. The dent tends to become planar but 
(a) $t=0$ to 10

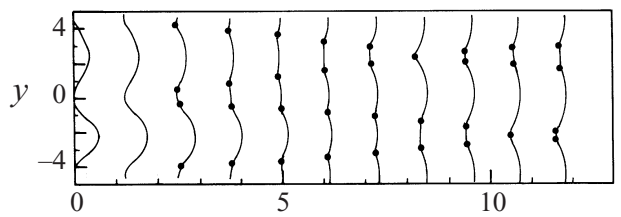

(b) $t=11$ to 20

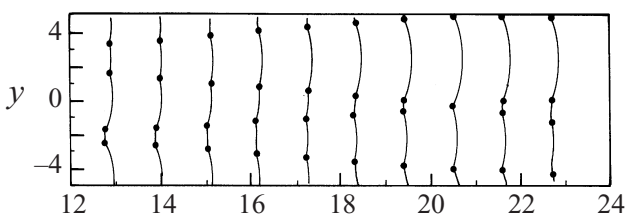

(c) $t=91$ to 100

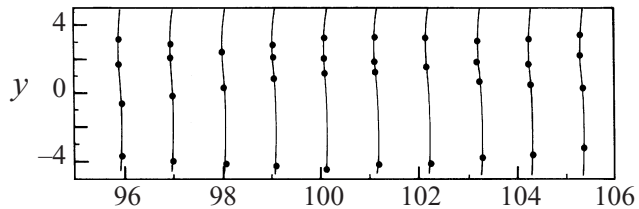

(d) $t=101$ to 110

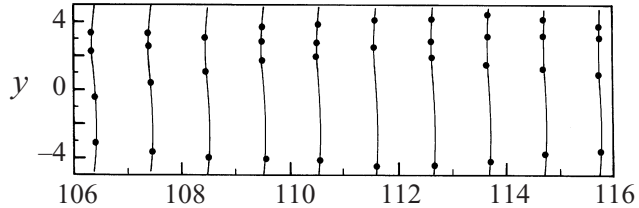

(e) $t=111$ to 120

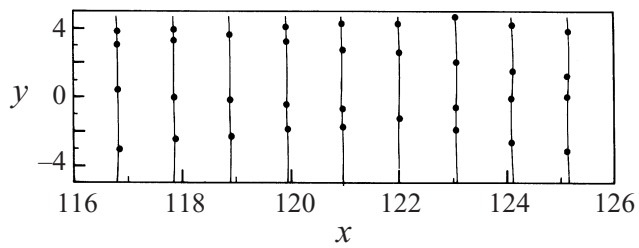

FIGURE 9. Long-term behaviour of a shock of periodic shape but far removed from a symmetric shape in each period.

in this process, the centre of the dent moves faster in such a way that the central portion becomes convex with the two kinks on its two sides contained in two dents as seen in figure 10. It has also been found that as the amplitude of the shock in the dent increases (i.e. $\beta$ decreases), the shock strength $M$ as well as the gradient of the density $N$ behind the shock increase (all measured at a fixed time, say $t=1$ ).

When the dent is quite deep (figure 11(b)), it appears that a kink has developed at the centre but a detailed study of the $(M, l)$ graph at $t=1$ shows that it is a pair of kinks which appears very close to the centre. At this time, the shock strength $M$ rises very rapidly at the centre to a value about 1.45 ; elsewhere it remains small and close to the initial value near 1.1. Soon the two kinks move away, and the shock develops a convex part at the centre separated by kinks from a concave part on either side.

The case of a single bulge is shown in figure 12: the bulge spreads initially and 
(a) $t=0$ to 10

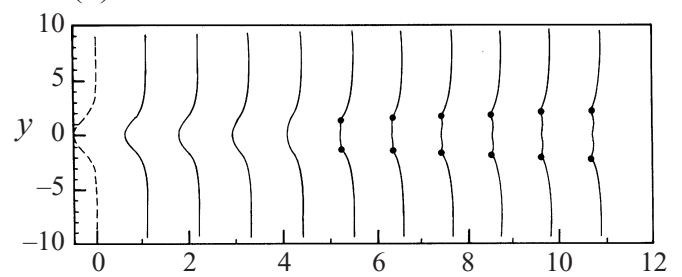

(b) $t=0$ to 10

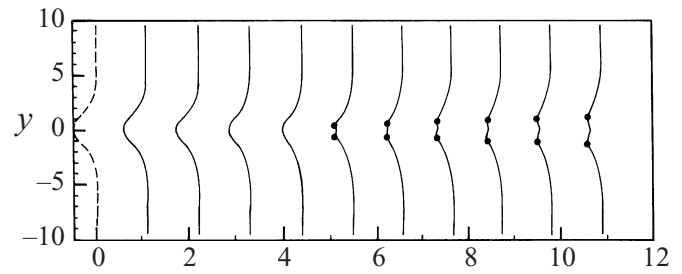

(c) $t=0$ to 10

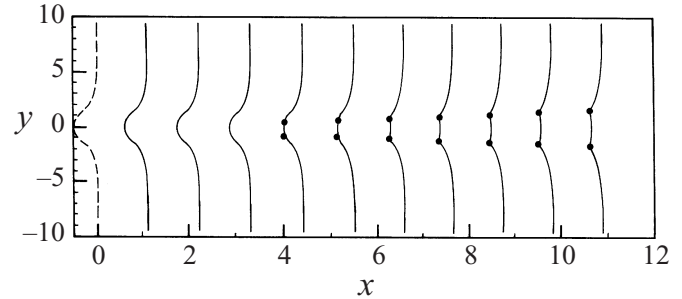

FIGURE 10. Successive positions of a shock front which initially has a smooth dent: $x=-\frac{1}{2} \exp \left(-y^{2} / 4\right), M_{0}=1.12 \exp \left(-\beta x^{2}\right), N_{0}=0.1$. (a) $\beta=-0.05,(b) \beta=0.02$, (c) $\beta=0$.

tends to become planar. Later on (after $t=16$ ), it becomes concave at the centre followed by two convex portions - one on either side. A pair of kinks (one on either side) is formed quite early and they move away from one another.

\section{Comparison with other theories}

6.1. Qualitative verification of the shape of the front obtained by DNS to support the kink theory

As already mentioned in the introduction, numerical results of Kevlahan (1996) provide enough justification of the validity of the present theory. We present here just one result of a numerical solution of the full gas dynamic equations.

The most important aspect of the results obtained by us is the resolution of the linear caustic and appearance of kinks on the shock front (also on nonlinear wavefronts by Prasad \& Sangeeta 1999). Numerical solution of Euler's equations of gas dynamics also leads to the same result: resolution of a linear caustic and appearance of kinks. We have done a computation for a curved piston problem with piston shape given by

$$
y^{2}=x / 2 \text { for }|y|<0.2, \quad y= \pm(x / 0.8+0.1) \text { for }|y|>2
$$

and initial piston velocity equal to 1 , piston acceleration also equal to 1 . The result is shown in figure 13. The numbers on the contours represent the values of the particle velocity in the $x$-direction on the contour. We first notice that the figure shows a 
(a) $t=0$ to 10

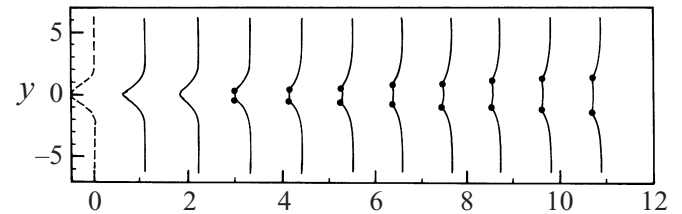

(b) $t=0$ to 10

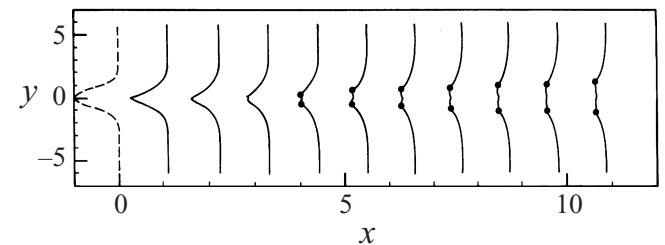

FiguRE 11. Successive positions of a shock front which initially has a smooth deep dent: (a) $x=-\frac{1}{2} \exp \left(-y^{2}\right), \quad M_{0}=1.12 \exp \left(-\beta x^{2}\right), \quad \beta=0.1, \quad N_{0}=0.1 ;$ (b) $x=-\exp \left(-y^{2}\right)$, $M_{0}=1.12 \exp \left(-\beta x^{2}\right), \beta=0.02, N_{0}=0.1$.

\section{(a) $t=0$ to 10}

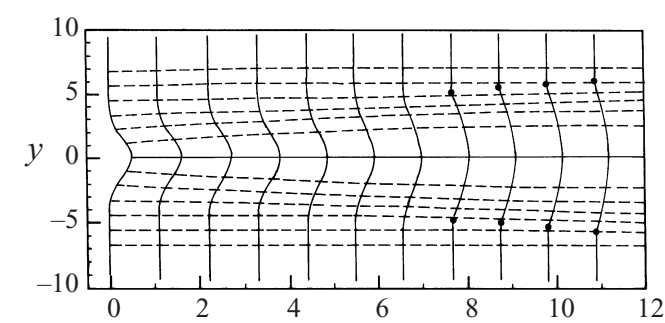

(b) $t=11$ to 20

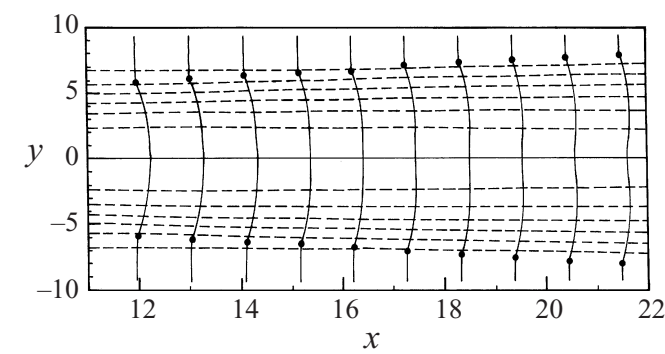

FIGURE 12. The initial front has a smooth bulge: $x=\frac{1}{2} \exp \left(-y^{2} / 4\right), \alpha=1.12, \beta=-0.05$ and $N_{0}=0.1$. Here also, a pair of kinks appears on the shock front and the two kinks move away from each other.

leading shock front with a well-defined kink across which the direction of the shock changes abruptly; on one side is the central disc and on the other side the wing. This clearly agrees with the qualitative picture of the shape of the shock front obtained by NTSD. In addition, there is a rapid compression region starting from the kink on the shock. The structure of the kink, as a shock front with continuously turning direction of the tangent, can be found on a length scale (along the shock) smaller than the radius of curvature of the piston and is discussed below. Numerical solution 


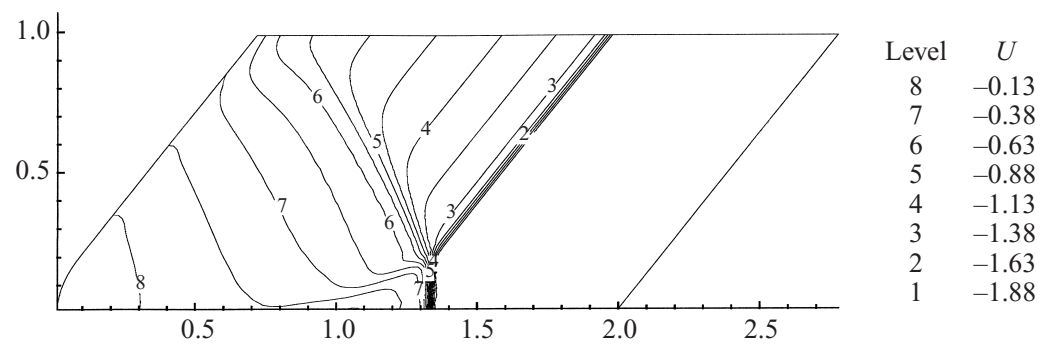

FIGURE 13. A shock front with a kink has been captured in the numerical solution of an accelerating curved piston problem using full gas dynamic equations in conservation form. The figure presents particle velocity contours (in the direction of the piston motion).

of the shock front using the shock ray theory of this paper takes very little computer time, say only $20 \%$ of the time of the results shown in figure 13 .

\subsection{Comparison with earlier theories}

The first and simplest theory of curved weak shock propagation to calculate the shock position is by fitting a weak shock in a one-parameter family of nonlinear waves moving along linear rays (Whitham 1956). This nonlinearization technique is equivalent to a systematic and formal derivation of a nonlinear ray theory for a general hyperbolic system by Choquet-Bruhat (1969) and for gas dynamic equations including many physical processes by Parker $(1969,1971)$.

Consider a hyperbolic system of $n$ conservation laws for

$$
\begin{gathered}
\boldsymbol{u}: \mathbb{R}^{m+1} \rightarrow \mathbb{R}^{n} \\
\left(\boldsymbol{f}^{(0)}(\boldsymbol{u})\right)_{t}+\sum_{\alpha=1}^{m}\left(\boldsymbol{f}^{(\alpha)}(\boldsymbol{u})\right)_{x_{\alpha}}=0 .
\end{gathered}
$$

A high-frequency asymptotic solution is of the form

$$
\boldsymbol{u}=\epsilon \boldsymbol{u}_{0}(t, \boldsymbol{x}, \theta)+\epsilon^{2} \boldsymbol{u}_{1}(t, \boldsymbol{x}, \theta)+\cdots, \quad \epsilon \rightarrow 0+,
$$

where the fast variable $\theta$ is related to the phase function $\phi(t, \boldsymbol{x})$ by

$$
\theta=\frac{\phi}{\epsilon}
$$

A straightforward substitution of (6.3) in (5.1) and equating coefficients of $\epsilon^{0}$ and $\epsilon^{1}$ to zero leads to the nonlinear theory of Choquet-Bruhat, Parker and Whitham (CPW theory).

This CPW-nonlinear ray theory for Euler's equations of gas dynamics for waves moving into a uniform medium at rest is described by ray equations (in suitable nondimensionalized variables) obtained by setting $w=0$ in the terms on the right-hand side of (2.1) and (2.2):

and a transport equation

$$
\begin{aligned}
& \frac{\mathrm{d} \boldsymbol{x}}{\mathrm{d} t}=\boldsymbol{n}, \\
& \frac{\mathrm{d} \boldsymbol{n}}{\mathrm{d} t}=0,
\end{aligned}
$$

$$
\left\{\frac{\mathrm{d}}{\mathrm{d} t}+\frac{\gamma+1}{2} \epsilon w\langle\boldsymbol{n} \cdot \nabla\rangle\right\} w=\Omega w
$$


where $w$ is the non-dimensional form of the perturbation in the density on the wave front, a scalar measure of the first term $\boldsymbol{u}_{0}$ in the expansion (6.3), and $\Omega$ is the mean curvature of the linear wavefront given by

$$
\Omega=-\frac{1}{2}\langle\nabla \cdot \boldsymbol{n}\rangle .
$$

Equations (6.5)-(6.6) give the rays and successive positions of a wavefront (chosen from the one-parameter family). These equations decouple from the transport equation (6.7), which is used to find the amplitude of the wave after the wavefront or the rays have been traced. A caustic appears whenever the initial wavefront is concave and amplitude becomes infinite, showing that the small-amplitude theory is no longer valid. A shock front fitted in the solution obtained by the above method cannot be continued in the caustic region where diffraction becomes important.

The diffraction effect, in the caustic region of the linear theory, takes place on a length scale of the order of $\epsilon^{1 / 2}$ in a direction transverse to the direction of the rays. To capture the diffraction phenomenon in a nonlinear wave, Hunter (1997) expanded the solution $\boldsymbol{u}$ of (6.2) in the form

$$
\boldsymbol{u}=\epsilon \boldsymbol{u}_{0}(t, \boldsymbol{x}, \theta, \eta)+\epsilon^{3 / 2} \boldsymbol{u}_{1}(t, \boldsymbol{x}, \theta, \eta)+\epsilon^{2} \boldsymbol{u}_{2}(t, \boldsymbol{x}, \theta, \eta)+O\left(\epsilon^{5 / 2}\right)
$$

where $\theta$ is related to the phase function $\phi$ by (5.3) and $\eta$ is a variable related to a new function $\psi(t, \boldsymbol{x})$ by

$$
\eta=\psi(t, \boldsymbol{x}) / \epsilon^{3 / 2}
$$

It turns out that $\psi$ is constant along the rays, confirming that the diffraction effects are captured on a length scale of order $\epsilon^{1 / 2}$ in the transverse direction. Expressing the amplitude of $\boldsymbol{u}_{0}$ in terms of a scalar $w$ and that of $\boldsymbol{u}_{1}$ in terms of $v$ and taking $\phi$ and $\psi$ to be linear functions of $t, x_{1}, \ldots, x_{n}$, it is possible to derive the two-dimensional Burgers equation

$$
\begin{gathered}
w_{t}+\left(\frac{1}{2} w^{2}\right)_{\theta}+v_{\eta}=0, \\
w_{\eta}-v_{\theta}=0 .
\end{gathered}
$$

Numerical solution of (6.11)-(6.12) by Hunter \& Brio (2000) (see also Hunter 1997) does show both the fish tail pattern of the very weak shock (see figures on pp. 242 and 243 of Hunter 1997) and the nonlinear Mach stem pattern for a moderately weak shock (see figures on pp. 240 and 241 of the same reference) observed by Sturtevant \& Kulkarni (1976). These are important and local solutions but it will be difficult to get them as a part of the global solution starting from a concave shock front, as done in this paper. What is important to note is that if we look at the shock front in the $(\xi, \eta)$-plane on a length scale of the order of $1 / \epsilon$, then the finer structure containing the continuously turning tangent of the figures on pp. 237, 239 and 240 (Hunter 1997) will reduce to a point - the leading shock will become a shock with a kink. Our theory does not give the flow behind the shock and hence the Mach stem or rapid compression region behind the two branches of the main shock cannot be obtained by our theory. Thus, we suggest that the Mach stem pattern solution of the two-dimensional Burgers equation gives the structure of a kink on a shock front. Of course, the two-dimensional Burgers equation has a limitation on its own validity for kinks which join the shocks on its two sides at angles which are not small. The two-dimensional Burgers equation (6.11)-(6.12) has been derived assuming that $\phi=0$ represents a plane shock. It will be interesting to study the original two-dimensional Burgers equation without an assumption that $\phi$ is a linear function of $x_{1}$ and $x_{2}$.

A second weakly nonlinear ray theory developed by Prasad since 1975 (see Prasad 
1994) is based on approximating the system (6.2) not in the neighbourhood of a linear wavefront but in the neighbourhood of the exact wavefront corresponding to the leading-order term $\epsilon u_{0}$ in (6.3) - we have been referring to this theory as WNLRT.

Numerical results using this theory are available in Prasad \& Sangeeta (1999). Derivation of the present NTSD from WNLRT in $\S 2$ (there also exists a completely independent derivation of NTSD) shows that NTSD and WNLRT are consistent. We shall discuss comparison with numerical results of WNLRT in the next section.

\subsection{Comparison with weakly nonlinear ray theory}

Now we discuss a comparison of the results of Prasad \& Sangeeta (1999) using the WNLRT and the shock ray theory presented in the previous section. Unlike equation (3.7), the transport equation for the WNLRT is homogeneous (i.e. without a term like $(M-1) N)$ and the second transport equation (3.8) is absent. This leads to a solution of a pair of conservation laws in the ray coordinate system

$$
\begin{aligned}
& (g \sin \theta)_{t}+(m \cos \theta)_{\xi}=0, \\
& (g \cos \theta)_{t}-(m \sin \theta)_{\xi}=0,
\end{aligned}
$$

where $g$ is a function of $m, m$ being the Mach number of the wavefront, $g$ the metric along it and $\theta$ the angle which the rays make with the $x$-axis. The shapes of the converging nonlinear wavefronts and initially sinusoidal or periodic wavefronts are almost the same as those obtained in this paper. Looking at these shapes alone, (and also those obtained by Kevlahan 1996), it is not possible to say more about the ultimate results as $t \rightarrow \infty$. The values of $m$ and $\theta$ approach the mean values of their distributions on the initial wavefronts. The computation also shows that in the periodic case, not only does $\lim _{t \rightarrow \infty}\left(m_{\max _{\xi \in \mathbb{R}}}(t)-m_{\min _{\xi \in \mathbb{R}}}(t)\right)$ tend to zero, as seen here, but also that $m_{\max }(t)$ and $m_{\min }(t)$ both approach constant values greater than 1 . Thus for a periodic wavefront, $m$ approaches a constant value uniformly as $t \rightarrow \infty$. The work of Glimm \& Lax (1970) shows that this asymptotic value is reached as $1 / t$. The numerical results in this paper show that the Mach number $M$ always decays to 1 (for $N>1$ ) and $\Theta$ tends to a constant as $t \rightarrow \infty$. For an initially periodic shock, $\Theta$ tends to zero (i.e. the shock front tends to become plane) and in the case of an initially concave shock front, $\Theta$ tends to zero on the central disc and a constant value on the wings. This implies that on a fixed ray $(\xi=$ constant), for large $t$, we may set $\Theta_{\xi}=0$ in (3.7) and (3.8). This gives

$$
M_{t}+N(M-1)=0
$$

and

$$
N_{t}+2 N^{2}=0 .
$$

Solving these two equations, we get the usual law of decay of shocks in the onedimensional Burgers equation

$$
M-1=\frac{M^{*}-1}{\left(2 N^{*}\left(t-t^{*}\right)+1\right)^{1 / 2}}, \quad N=\frac{N^{*}}{2 N^{*}\left(t-t^{*}\right)+1},
$$

where $M^{*}$ and $N^{*}$ are the values of $M$ and $N$ at $t=t^{*}$ and it is assumed that this ray does not pass through a kink during the time interval $\left(t, t^{*}\right)$. This shows that the shock strength $M-1$ tends to zero as $O\left(t^{-1 / 2}\right)$ and $N$ tends to zero as $O\left(t^{-1}\right)$. This is a major difference from the results of WNLRT. A comparison of the results obtained from NTSD and WNLRT is presented in figures 14 and 15. 

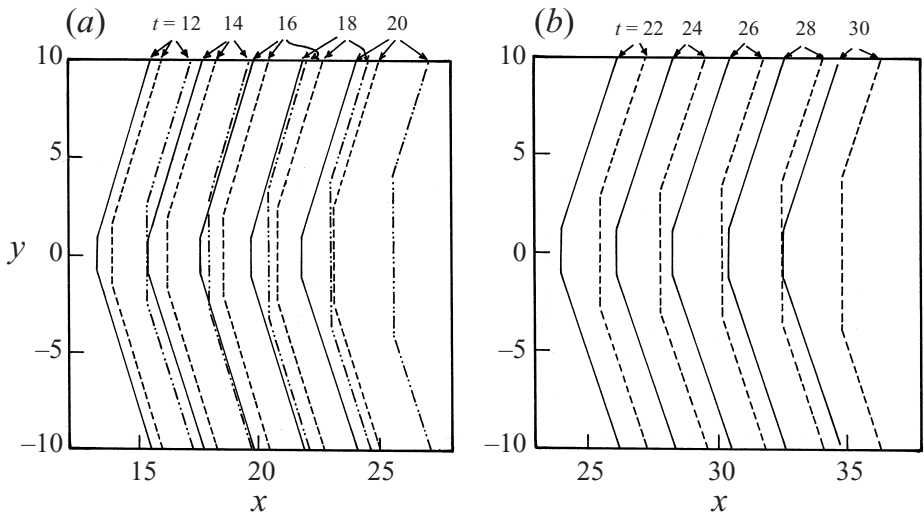

FIGURE 14. Comparison of the NTSD and Whitham's theory (a) Comparison of the successive positions of the shock fronts and nonlinear wavefronts starting with the same initial front: $y^{2}=8 x$, for $|y|<1$, and the same amplitude distribution on it $(\mu=1 / 6): \alpha=1.1, \beta=0$. Solid curves: shock front by NTSD $\left(N_{0}=0.15\right), M_{0}=1+0.6 \mu$. Dotted curves: shock front by Whitham's shock dynamics $M_{0}=1+0.6 \mu$. Broken curves: nonlinear wavefronts (Prasad and Sangeeta) $M_{0}=1+1.2 \mu$. (b) The comparison in $(a)$ continues for larger times. The size of the central disc varies considerably and is much bigger in the case of Whitham's front.

(a)

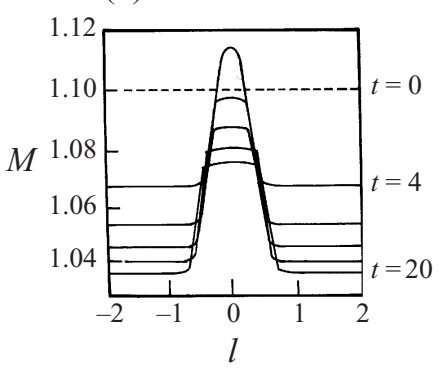

(b)

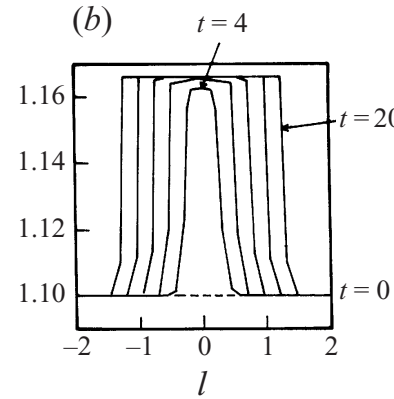

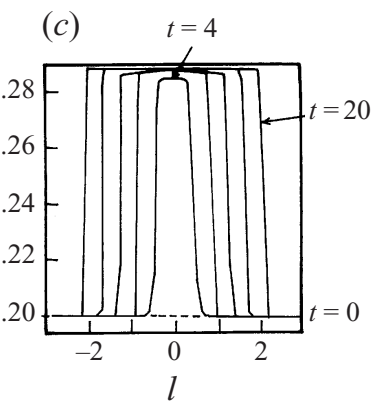

FiguRE 15. Comparison of the Mach number distribution on the fronts (at times $0,2,4, \ldots, 20$ ) with the same amplitude (excess density $\mu$ ) distribution on the initial front for the case represented in figure 14 with $(a)$ NTSD, $(b)$ Whitham's theory, $(c)$ WNLRT. The Mach number at the centre of the fronts rises considerably in all the cases but it becomes constant on the central disc for the Whitham's shock front and nonlinear wavefront. As seen in all previous cases with $N_{0}>0$, the Mach number (and hence the wave amplitude) decreases with time on all parts of the shock front by NTSD.

\subsection{Comparison with Whitham's theory}

As mentioned in the introduction, Whitham $(1957,1959)$ proposed a theory of shock dynamics, which is very simple from the point of view of applications. Even though the theory does not properly take into account the interaction of the shock with the flow behind it, it has given good results for the successive positions of a shock and the kinks in some cases (Henshaw, Smyth \& Schwendeman 1986; Schwendeman 1989) especially for strong shocks. For a weak shock, the equations of Whitham's shock dynamics expressed in terms of the Mach number of the shock, are exactly the same as the differential form of the equations of nonlinear ray theory (Prasad 1993; Prasad $\&$ Sangeeta 1999), but the relation of the Mach number $M$ of a shock front and $m$ of a nonlinear wavefront to the perturbation $w$ of WNLRT (or the corresponding value 
$\mu$ in this paper) is different. We first note that $\mu$ and non-dimensional $w$ are related

$$
\left.w\right|_{\text {shock }}=\mu \text {. }
$$

The relations between $M, m$ and $w$ (or $\mu$ ) are

$$
M=1+\epsilon \frac{\gamma+1}{4} \mu, \quad m=1+\epsilon \frac{\gamma+1}{2} w .
$$

For comparison of our results in this paper with Whitham's shock dynamics and WNLRT, we have taken a few cases of a converging shock and prescribed the same value of $w($ or $\mu)$ on the initial wavefront in all three cases. In addition, we shall have to prescribe $N$ for the NTSD used in this paper. The use of our conservation form of the equations makes calculation by Whitham's theory very simple.

The results show that in early stages, the nonlinear wavefront by WNLRT is a little ahead of the shock front by NTSD and Whitham's theory, but all three differ considerably as $t$ increases. The nonlinear wavefront has moved so far away from the other two that it has not been shown after $t=20$ in figure 14. It is true that qualitatively the geometry of the fronts is similar but Whitham's theory gives a bigger shock disc. However, the most striking result seen from figure 15, is that the shock strength by Whitham's theory attains a constant value on the disc and the wings, whereas that obtained by the NTSD decreases with time. This causes the former to move ahead of the latter, and the separation between the kinks keeps on increasing with time. This, of course, is expected as Whitham's theory does not properly take into account the interaction of the shock with the nonlinear waves which catch up with it from behind.

Figure 14 shows that at time $t=30$ the central disc of Whitham's shock has moved about $10 \%$ ahead of the distance travelled by the shock calculated by NTSD. Figure 15 shows that the Mach number at the centre of the fronts rises considerably in all three cases but it becomes constant on the central disc of Whitham's shock front and another much higher constant on the nonlinear wavefront. As seen in all the previous cases with $N_{0}>0$, the Mach number (and hence the wave amplitude) decreases with time on all shock rays of NTSD. At $t=20$ the wave amplitude in terms of $M-1$ on the shock disc by NTSD has become less than half of the maximum value 1.166 attained at the centre of the shock by Whitham's theory. The shock disc in Whitham's shock increases in size much faster.

\subsection{Corrugational stability and persistence of a kink}

Corrugational stability, by which we mean that a plane shock is stable, seems to have been discussed first by Gardner \& Kruskal (1964) in the case of a magnetohydrodynamic shock. Though Whitham (1974) uses his theory of shock dynamics to discuss this problem qualitatively, he deduces important results that (i) a perturbation like a single bulge or dent will decay as $t^{-1 / 2}$ and (ii) a perturbation like sinusoidal shape will decay as $t^{-1}$. Anile \& Russo obtained an exact stability criterion in 1986 (see Anile et al. 1993 for corrugational stability). The WNLRT and the NTSD are ideally suited to discuss the corrugational stability of a nonlinear wavefront and a shock front respectively. In the case of a perturbation of a plane front into a periodic front (like the one discussed in $\S 5.2$ ), we can use results like those available in Glimm \& Lax (1970) to prove rigorously that the front tends to be plane as $t^{-1}$. However, the Glimm-Lax assumption that 'interaction of two shocks of the same family always produces a shock of the same family plus a rarefaction wave of the opposite family' is not satisfied even in the simpler system of the pair of conservation laws of the 
WNLRT (a detailed discussion of this will appear in a future publication by Baskar $\&$ Prasad). In spite of this, extensive numerical computation by Prasad \& Sangeeta (1999) with WNLRT shows that a plane nonlinear wavefront is stable. Computations in this paper indicate corrugational stability of a plane shock front. Here we have some additional results with dents and bulges on plane shock fronts - all these results point to the corrugational stability.

The corrugational stability is a result of genuine nonlinearity in the characteristic fields with characteristic velocities $\pm \sqrt{(M-1) / 2 G^{2}}$ of the system (3.6)-(3.8). The shocks in the $(\xi, t)$-plane, which are mapped into kinks, seem to cause dissipation of the kinetic energy. Further mathematical investigation in this dissipation is required. The equations of WNLRT are homogeneous but one of the equations of the NTSD namely equation (3.16) has a source term. For $N>0$, as seen from (6.17), this additional term causes the shock amplitude $M-1$ to tend to zero. This is typical of a plane shock in gas dynamics (as can be seen from the model equation $u_{t}+\left(\frac{1}{2} u^{2}\right)_{x}=0$ ). Combining both these features, we interpret our results as the genuine nonlinearities in the two characteristic fields cause decay of the shock amplitude and perturbation in its geometrical shape leading to corrugational stability of a shock but in addition to this the non-homogeneous term in the equation (3.16) causes the shock strength to tend to zero when $N>0$. When both these effects are included, the law of decay as pointed out by Whitham may have to be modified. We need further investigation on this.

The persistence of kinks is an interesting phenomenon, which we observe from the long time computation. As we have seen in all the cases considered by us, a kink may appear on an initially smooth shock front, but once it is formed it persists till it meets another kink. The persistence of a kink follows from the similar property of a shock in a genuinely nonlinear characteristic field: a shock, once formed cannot terminate at a finite distance in the $(\xi, t)$-plane. A proof of this statement is available in Prasad $(1993, \S 1.5)$. We further notice that interaction of a pair of shocks (whether the shocks belong to the same characteristic field or different characteristic fields) always produces another pair of shocks (Baskar \& Prasad-under preparation). Thus the number of kinks on the shock front always remains the same after some time when no new kinks are produced.

The authors express sincere thanks to Dr K. S. Ravichandran for valuable help in directing us towards the appropriate numerical methods for the solution of full gas dynamic equations leading to figure 13 . We would also like to thank S. Baskar for providing figure 1 and for the precious time he spent in improving this paper.

\section{REFERENCES}

Anile, A. M., Hunter, J. K., Pantano, P. \& Russo, G. 1993 Ray Methods for Nonlinear Waves in Fluids and Plasmas. Pitman.

Anile, A. M. \& Russo, G. 1986 Corrugation stability for plane relativistic shock waves. Phys. Fluids 29, 2847-2852.

Brio, M. \& Hunter, J. K. 1992 Mach reflection for the two dimensional Burgers equation. Physica D 60, 194-207.

Brio, M. \& Hunter, J. K. 1994 A von Neumann reflection for the 2-d Burgers equation. Proc. Symp. on Applied Mathematics, vol. 48, pp. 265-268.

Choquet-Bruhat, Y. 1969 Ondes asymptotiques et approchees pour de systems d'equations aux deriveés partialles nonlinéaires. J. Math Pures et Appl. 48, 117-158.

Cockburn, B., SAn Yih-Lin \& Shu, C. W. 1989 TVB Runge-Kutta local projection discontinuous Galerkin finite element method for conservation laws III: One dimensional systems. J. Comput. Phys. 84, 90-113. 
Collela, P. \& Henderson, L. F. 1990 The von Neumann paradox for the diffraction of weak shock waves. J. Fluid Mech. 213, 71-94.

Gardner, C. S. \& Kruskal, M. D. 1964 Stability of plane magnetohydrodynamic shocks. Phys. Fluids 7, 700-706.

Giles, M., Prasad, P. \& Ravindran, R. 1996 Conservation form of equations of three dimensional front propagation. Preprint. Dept. Maths, Indian Institute of Science.

Glimm, J. \& LaX, P. D. 1970 Decay of solutions of nonlinear hyperbolic conservation laws. Am. Math. Soc. Memoir 101.

GrinfeL'D, M. A. 1978 Ray method for calculating the wave front intensity in nonlinear elastic material. J. Appl. Math. Mech. 42, 958-977.

Henshaw, W. D., Smyth, N. F. \& Schwendeman, D. W. 1986 Numerical shock propagation using geometrical shock dynamics. J. Fluid Mech. 171, 519-545.

Hunter, J. K. 1997 Nonlinear wave diffraction. In Geometrical Optics and Related Topics (ed. F. Colombini \& N. Lerner), pp. 221-243. Birkhauser.

Hunter, J. K. \& Brio, M. 2000 Weak shock reflection. J. Fluid Mech. 410, 235-261.

Kevlahan, N. K.-R. 1994 Structure and shocks in turbulence. PhD thesis, University of Cambridge.

KeVlahan, N. K.-R. 1996 The propagation of weak shocks in non-uniform flows. J. Fluid Mech. 327, 167-197.

Lazarev, M. P., Prasad, P. \& Singh, S. K. 1995 An approximate solution of one dimensional piston problem. Z. Angew. Math. Phys. 46, 752-771.

LeVeque, R. J. \& YeE, H. C. 1990 A study of numerical methods for hyperbolic conservation laws with stiff source term. J. Comput. Phys. 86, 187-210.

Maslov, V. P. 1980 Propagation of shock waves in an isentropic nonviscous gas. J. Soviet Maths 13, 119-163.

MonicA, A. 1999 Propagation of a curved weak shock front. PhD thesis, Indian Institute of Science, Bangalore.

Morton, K. W., Prasad, P. \& Ravindran, R. 1992 Conservation form of nonlinear ray equations. Tech. Rep. 2. Dept. of Maths, Indian Institute of Science.

PARKer, D. F. 1969 Nonlinearity, relaxation and diffusion in acoustics and ultrasonics. J. Fluid Mech. 39, 793-815.

Parker, D. F. 1971 An asymptotic theory for oscillatory nonlinear signals. J. Inst. Maths Applics. 7, 92-110.

Prasad, P. 1975 Approximation of perturbation equations in a quasilinear hyperbolic system in the neighbourhood of a bicharacteristic. J. Math Anal. Applics. 50, 470-482.

Prasad, P. 1982 Kinematics of a multi-dimensional shock of arbitrary strength in an ideal gas. Acta Mech. 45, 163-176.

Prasad, P. 1987 Extension of Huygen's construction of a wavefront to a nonlinear wavefront and a shock front. Current Sci. 56, 50-54.

Prasad, P. 1993 Propagation of a Curved Shock and Nonlinear Ray Theory. Longman.

Prasad, P. 1994 A nonlinear ray theory. Wave Motion 20, 21-31.

PRASAD, P. 1995 Formation and propagation of singularities on a nonlinear wavefront and a shock front. J. Indian Inst. Sci. 75, 517-535.

Prasad, P. 2000 An asymptotic derivation of weakly nonlinear ray theory. Proc. Indian Acad. Sci. (Math. Sci.) 110, 1-17.

Prasad, P. 2001 Nonlinear Hyperbolic Waves in Multi-dimensions. CRC Press.

Prasad, P., Ravindran, R. \& SaU, A. 1991 On the characteristic rule for shocks. Appl. Math. Lett. 4, 5-8.

Prasad, P. \& SANGeEta, K. 1999 Numerical simulation of converging nonlinear wavefronts. J. Fluid Mech. 385, 1-20.

Ravindran, R. \& Prasad, P. 1993 On an infinite system of compatibility conditions along a shock ray. Q. J. Appl. Maths Mech. 46, 131-140.

Schwendeman, D. W. 1988 Numerical shock propagation in non-uniform media. J. Fluid Mech. 188, 383-410.

SHU, C. W. 1987 TVB uniformly higher order schemes for conservation laws. Maths Comput. 49, $105-121$ 
Singh, S. K. \& Singh, V. P. 1999 Approximate solution of 2-d piston problem. Z. Angew Math. Phys. 50, 1-16.

SRINIVASAN, R. \& Prasad, P. 1985 On the propagation of a multi-dimensional shock of arbitrary strength. Proc. Indian Acad. Sci. (Math. Sci.) 94, 27-42.

Strang, G. 1968 On the construction and comparison of difference schemes. SIAM J. Numer. Anal. 5, 506-517.

Sturtevant, B. \& Kulkarni, V. A. 1976 The focusing of weak shock waves. J. Fluid Mech. 73, 651-671.

TABAK, E. G. \& Rosales, R. R. 1994 Focusing of weak shock waves and the von Neumann paradox of oblique shock reflection. Phys. Fluids 6, 1874-1892.

Whitham, G. B. 1956 On the propagation of weak shock waves. J. Fluid Mech. 1, 290-318.

Whitham, G. B. 1957 A new approach to problems of shock dynamics. Part 1. Two dimensional problems. J. Fluid Mech. 2, 145-171.

Whitham, G. B. 1959 A new approach to problems of shock dynamics. Part 2. Three dimensional problems. J. Fluid Mech. 5, 369-386.

Whiтнам, G. B. 1974 Linear and Nonlinear Waves. Wiley. 